\title{
Geometry of the Moment Map for Representations of Quivers
}

\section{WILLIAM CRAWLEY-BOEVEY}

Department of Pure Mathematics, University of Leeds, Leeds LS2 9JT, U.K. e-mail:w.crawley-boevey@leeds.ac.uk

(Received: 2 November 1999; accepted in final form: 18 May 2000)

Abstract. We study the moment map associated to the cotangent bundle of the space of representations of a quiver, determining when it is flat, and giving a stratification of its Marsden-Weinstein reductions. In order to do this we determine the possible dimension vectors of simple representations of deformed preprojective algebras. In an appendix we use deformed preprojective algebras to give a simple proof of much of Kac's Theorem on representations of quivers in characteristic zero.

Mathematics Subject Classifications (2000). Primary 16G20, 53D20.

Key words. quiver representation, moment map, preprojective algebra.

\section{Introduction}

Let $K$ be an algebraically closed field and let $Q$ be a quiver with vertex set $I$. Representations of $Q$ of dimension vector $\alpha \in \mathbb{N}^{I}$ are given by elements of the space

$$
\operatorname{Rep}(Q, \alpha)=\bigoplus_{a \in Q} \operatorname{Mat}\left(\alpha_{h(a)} \times \alpha_{t(a)}, K\right)
$$

where $h(a)$ and $t(a)$ are the head and tail vertices of an arrow $a \in Q$; isomorphism classes correspond to orbits of the group

$$
\mathrm{G}(\alpha)=\left(\prod_{i \in I} \mathrm{GL}\left(\alpha_{i}, K\right)\right) / K^{*}
$$

acting by conjugation. Using the trace pairing there is an identification of the cotangent bundle

$$
\mathrm{T}^{*} \operatorname{Rep}(Q, \alpha) \cong \operatorname{Rep}(\bar{Q}, \alpha)
$$

where $\bar{Q}$ is the double of $Q$, obtained by adjoining a reverse arrow $a^{*}: j \rightarrow i$ for each arrow $a: i \rightarrow j$ in $Q$. 
We consider the moment map $\mu_{\alpha}: \operatorname{Rep}(\bar{Q}, \alpha) \rightarrow \operatorname{End}(\alpha)_{0}$ defined by

$$
\mu_{\alpha}(x)_{i}=\sum_{\substack{a \in Q \\ h(a)=i}} x_{a} x_{a^{*}}-\sum_{\substack{a \in Q \\ t(a)=i}} x_{a^{*}} x_{a}
$$

where

$$
\operatorname{End}(\alpha)_{0}=\left\{\left(\theta_{i}\right) \mid \sum_{i \in I} \operatorname{tr}\left(\theta_{i}\right)=0\right\} \subseteq \operatorname{End}(\alpha)=\bigoplus_{i \in I} \operatorname{Mat}\left(\alpha_{i}, K\right) .
$$

If one uses the trace pairing to identify $\operatorname{End}(\alpha)_{0}$ with the dual of the Lie algebra of $\mathrm{G}(\alpha)$, then this is a moment map in the usual sense. (Identifying $\operatorname{Rep}(\bar{Q}, \alpha)$ with its tangent space at any point, the natural symplectic form on the cotangent bundle corresponds to the form

$$
\omega(x, y)=\sum_{a \in Q}\left(\operatorname{tr}\left(x_{a} y_{a^{*}}\right)-\operatorname{tr}\left(x_{a^{*}} y_{a}\right)\right)
$$

on $\operatorname{Rep}(\bar{Q}, \alpha)$. Now if $\theta \in \operatorname{End}(\alpha)$, and $f: \operatorname{Rep}(\bar{Q}, \alpha) \rightarrow K$ is defined by $f(x)=$ $\sum_{i} \operatorname{tr}\left(\theta_{i} \mu_{\alpha}(x)_{i}\right)$, then $\mathrm{d} f_{x}(y)=\omega([\theta, x], y)$ for $x, y \in \operatorname{Rep}(\bar{Q}, \alpha)$, where $[\theta, x]$ is defined by $[\theta, x]_{a}=\theta_{h(a)} x_{a}-x_{a} \theta_{t(a)}$ for any $a \in \bar{Q}$.)

Now the elements of $\operatorname{End}(\alpha)_{0}$ which are invariant under $\mathrm{G}(\alpha)$ acting by conjugation are those whose components are scalar matrices. We identify them with the $\lambda \in K^{I}$ which have $\lambda \cdot \alpha=\sum_{i \in I} \lambda_{i} \alpha_{i}$ equal to zero. In this paper we study the fibres $\mu_{\alpha}^{-1}(\lambda)$ and the quotients $\mu_{\alpha}^{-1}(\lambda) / / \mathrm{G}(\alpha)$. These are Marsden-Weinstein reductions [15], except that we work with schemes rather than manifolds.

This moment map has been considered before. Kronheimer [11] constructed the Kleinian singularities and their deformations in this way from the extended Dynkin quivers (see also [2, 5]). Later, Lusztig [14, Section 12] used the nilpotent cone of $\mu_{\alpha}^{-1}(0)$ in his geometric construction of the negative part of the quantum group of type $Q$, for any quiver $Q$ without loops. Finally Nakajima [16-18] used the moment map to define some quiver varieties and used these in a geometric construction of integrable representations of Kac-Moody Lie algebras. In the first of his papers he used hyper-Kähler quotients to define a family $\mathfrak{M}_{\zeta}$, and this family includes $\mu_{\alpha}^{-1}(\lambda) / / \mathrm{G}(\alpha)$ with $K=\mathbb{C}$ by [16, Theorem 3.1]. In his later papers he used geometric invariant theory quotients, and $\mu_{\alpha}^{-1}(0) / / \mathrm{G}(\alpha)$ appears as the variety $\mathfrak{M}_{0}(\mathbf{v}, 0)$ in $[18, \S 3]$.

Kac $[7,8]$ has shown that the dimension vectors of indecomposable representations of $Q$ are exactly the positive roots for $Q$, and that the number of parameters of indecomposable representations of dimension $\alpha$ is given by the function

$$
p(\alpha)=1+\sum_{a \in Q} \alpha_{t(a)} \alpha_{h(a)}-\alpha \cdot \alpha,
$$

where $\alpha \cdot \alpha=\sum_{i \in I} \alpha_{i}^{2}$. After some preliminaries in Sections 2 and 3, we use Kac's 
Theorem in Section 4 to compute the dimension of $\mu_{\alpha}^{-1}(\lambda)$ and then use his 'canonical decomposition' to prove the following result.

THEOREM 1.1. If $\alpha \in \mathbb{N}^{I}$ then the following are equivalent

(1) $\mu_{\alpha}$ is a flat morphism.

(2) $\mu_{\alpha}^{-1}(0)$ has dimension $\alpha \cdot \alpha-1+2 p(\alpha)$.

(3) $p(\alpha) \geqslant \sum_{t=1}^{r} p\left(\beta^{(t)}\right)$ for any decomposition $\alpha=\beta^{(1)}+\cdots+\beta^{(r)}$ with the $\beta^{(t)}$ positive roots.

(4) $p(\alpha) \geqslant \sum_{t=1}^{r} p\left(\beta^{(t)}\right)$ for any decomposition $\alpha=\beta^{(1)}+\cdots+\beta^{(r)}$ into nonzero $\beta^{(t)} \in \mathbb{N}^{I}$.

The deformed preprojective algebra introduced by M. P. Holland and the author [5] (see also [3]) is the algebra defined for $\lambda \in K^{I}$ by

$$
\Pi^{\lambda}=K \bar{Q} /\left(\sum_{a \in Q}\left[a, a^{*}\right]-\sum_{i \in I} \lambda_{i} e_{i}\right)
$$

where $K \bar{Q}$ is the path algebra of $\bar{Q}$, the trivial path at vertex $i$ is denoted $e_{i}$, and $\left[a, a^{*}\right]$ is the commutator $a a^{*}-a^{*} a$.

Clearly if $\lambda \in K^{I}$ and $\lambda \cdot \alpha=0$, then $\mu_{\alpha}^{-1}(\lambda)$ is identified with the space of representations of $\Pi^{\lambda}$ of dimension vector $\alpha$. Now the closed orbits of $G(\alpha)$ on $\operatorname{Rep}(\bar{Q}, \alpha)$ correspond to isomorphism classes of semisimple representations of $\bar{Q}$ of dimension $\alpha$. (For example, take $\theta=0$ in [9, Proposition 3.2].) Thus the closed orbits of $\mathrm{G}(\alpha)$ on $\mu_{\alpha}^{-1}(\lambda)$ correspond to isomorphism classes of semisimple representations of $\Pi^{\lambda}$ of dimension $\alpha$. Of these, the orbits on which $G(\alpha)$ acts freely are those corresponding to a simple representation of $\Pi^{\lambda}$. Our main result is as follows.

THEOREM 1.2. For $\lambda \in K^{I}$ and $\alpha \in \mathbb{N}^{I}$ the following are equivalent

(1) There is a simple representation of $\Pi^{\lambda}$ of dimension vector $\alpha$

(2) $\alpha$ is a positive root, $\lambda \cdot \alpha=0$, and $p(\alpha)>\sum_{t=1}^{r} p\left(\beta^{(t)}\right)$ for any decomposition $\alpha=\beta^{(1)}+\cdots+\beta^{(r)}$ with $r \geqslant 2$ and $\beta^{(t)}$ a positive root with $\lambda \cdot \beta^{(t)}=0$ for all $t$.

In this case $\mu_{\alpha}^{-1}(\lambda)$ is a reduced and irreducible complete intersection of dimension $\alpha \cdot \alpha-1+2 p(\alpha)$, and the general element of $\mu_{\alpha}^{-1}(\lambda)$ is a simple representation of $\Pi^{\lambda}$.

The special case $\lambda=0$ answers some questions of Nakajima. In [17, Problem 4.6], in the situation where $Q$ has no loops, Nakajima asks whether if $Q$ is connected and non-Dynkin then $\Pi^{0}$ has a simple representation which is not one-dimensional. This is true, for in Theorem 1.2 one can take $\alpha$ to be any minimal imaginary root. In [18, Question after Lemma 4.9], he asks which elements of the fundamental region are dimension vectors of simple representations of $\Pi^{0}$. The answer is given by Theorems 1.2 and 8.1 . 
Henceforth we write $\Sigma_{\lambda}$ for the set of $\alpha$ satisfying the conditions in part (2) of Theorem 1.2. In Section 5 we study the set $\Sigma_{\lambda}$, and provide another characterization of it. In Section 6 we use Kac's Theorem again to prove that $\mu_{\alpha}^{-1}(\lambda)$ is irreducible of dimension $\alpha \cdot \alpha-1+2 p(\alpha)$ for $\alpha \in \Sigma_{\lambda}$. We then use Schofield's theory of general representations of quivers to show that the general element of $\mu_{\alpha}^{-1}(\lambda)$ is a simple representation. This proves $(2) \Longrightarrow(1)$. The implication $(1) \Longrightarrow(2)$ is more complicated and is proved in Sections 7 to 10 .

If $\alpha \in \Sigma_{\lambda}$, how many simple representations of dimension $\alpha$ are there? The $\mathrm{G}(\alpha)$-orbit of a simple representation has dimension $\alpha \cdot \alpha-1$. Thus if $\alpha$ is a real root (so $p(\alpha)=0$ ), there is a unique simple representation up to isomorphism, while if $\alpha$ is an imaginary $\operatorname{root}($ so $p(\alpha)>0$ ), there are infinitely many non-isomorphic simple representations.

Now suppose that $K$ has characteristic zero. In Section 11 we study the affine quotient schemes $\mu_{\alpha}^{-1}(\lambda) / / \mathrm{G}(\alpha)$. Recall that the points of this quotient are in one-to-one correspondence with the closed orbits, so with isomorphism classes of semisimple representations of $\Pi^{\lambda}$ of dimension $\alpha$. Given a semisimple representation $X$, we can decompose it into its simple components $X=X_{1}^{\oplus k_{1}} \oplus \cdots \oplus$ $X_{r}^{\oplus k_{r}}$ where the $X_{t}$ are non-isomorphic simples. If $\beta^{(t)}$ is the dimension vector of $X_{t}$, we say that $X$ has representation type $\tau=\left(k_{1}, \beta^{(1)} ; \ldots ; k_{r}, \beta^{(r)}\right)$. For $\tau$ to occur as the representation type of a semisimple representation of dimension $\alpha$, clearly one must have $\alpha=k_{1} \beta^{(1)}+\cdots+k_{r} \beta^{(r)}$ and $\beta^{(t)} \in \Sigma_{\lambda}$ for all $t$. In addition, although the $\beta^{(t)}$ need not be distinct, any real root can occur as at most one of the $\beta^{(t)}$.

THEOREM 1.3. If $\tau$ is a representation type, then the set of semisimple representations of type $\tau$ is an irreducible locally closed subset of $\mu_{\alpha}^{-1}(\lambda) / / \mathrm{G}(\alpha)$ of dimension $\sum_{t=1}^{r} 2 p\left(\beta^{(t)}\right)$.

This has the following consequence.

COROLLARY 1.4. If $\lambda \in K^{I}$ and $\alpha \in \Sigma_{\lambda}$ then $\mu_{\alpha}^{-1}(\lambda) / / \mathrm{G}(\alpha)$ is a reduced and irreducible scheme of dimension $2 p(\alpha)$.

Finally, in an appendix we show how deformed preprojective algebras can be used to give a simple proof of much of Kac's Theorem in case the base field has characteristic zero. In particular, we give an explicit construction of the indecomposable representations whose dimension vector is a real root.

Preliminary versions of these results (with $\lambda=0$ ) were first announced at a conference on Geometry and Quivers in Hamburg in November 1996. I should like to thank the organisers O. Riemenschneider and P. Slodowy for inviting me to attend the meeting. I would also like to thank M. P. Holland for some useful discussions.

Remarks added in April 2000 (after writing the paper [4]). We would like to explain some additional applications of the results in this paper to the study of Nakajima's quiver varieties. 
Let $Q_{0}$ be a quiver with vertex set $I$. In case $Q_{0}$ has no oriented cycles this is to correspond to an orientation $\Omega$ of a graph $(I, E)$ as in [18, Section 3.1]. For $\mathbf{v}, \mathbf{w} \in \mathbb{N}^{I}$, let $\mathbf{M}(\mathbf{v}, \mathbf{w})$ be the space

$$
\operatorname{Rep}\left(\overline{Q_{0}}, \mathbf{v}\right) \oplus \bigoplus_{k \in I} \operatorname{Mat}\left(\mathbf{v}_{k} \times \mathbf{w}_{k}, K\right) \oplus \bigoplus_{k \in I} \operatorname{Mat}\left(\mathbf{w}_{k} \times \mathbf{v}_{k}, K\right) .
$$

There is a natural action of the group $G_{\mathbf{v}}=\prod_{k \in I} \mathrm{GL}\left(\mathbf{v}_{k}, K\right)$ and a moment map

$$
\mu: \mathbf{M}(\mathbf{v}, \mathbf{w}) \rightarrow \bigoplus_{k \in I} \operatorname{Mat}\left(\mathbf{v}_{k}, K\right)
$$

whose $k$-th component sends $(B, i, j)$ to

$$
\sum_{\substack{a \in Q_{0} \\ h(a)=k}} B_{a} B_{a^{*}}-\sum_{\substack{a \in Q_{0} \\ t(a)=k}} B_{a^{*}} B_{a}+\sum_{k \in I} i_{k} j_{k} .
$$

One of the spaces that Nakajima considers is

$$
\mathfrak{M}_{0}(\mathbf{v}, \mathbf{w})=\mu^{-1}(0) / / G_{\mathbf{v}} .
$$

Let $Q$ be the quiver obtained from $Q_{0}$ by adjoining a new vertex $\infty$ and $\mathbf{w}_{k}$ arrows from $\infty$ to $k$ for each $k \in I$; let $\alpha$ be the dimension vector for $Q$ whose restriction to $I$ is equal to $\mathbf{v}$ and with $\alpha_{\infty}=1$. By dividing the matrices in $\operatorname{Mat}\left(\mathbf{v}_{k} \times \mathbf{w}_{k}, K\right)$ into their columns, and the matrices in $\operatorname{Mat}\left(\mathbf{w}_{k} \times \mathbf{v}_{k}, K\right)$ into their rows, one can identify

$$
\mathbf{M}(\mathbf{v}, \mathbf{w}) \cong \operatorname{Rep}(\bar{Q}, \alpha), \quad G_{\mathbf{v}} \cong \mathrm{G}(\alpha) .
$$

Moreover, $\mu$ corresponds to the usual moment map $\mu_{\alpha}$, so we have

$$
\mathfrak{M}_{0}(\mathbf{v}, \mathbf{w}) \cong \mu_{\alpha}^{-1}(0) / / \mathrm{G}(\alpha) .
$$

Thus the set $\mathfrak{M}_{0}^{\text {reg }}(\mathbf{v}, \mathbf{w})$ of $[18, \S 3 . v]$ is nonempty if and only if $\alpha \in \Sigma_{0}$.

The other space that Nakajima considers is the quiver variety

$$
\mathfrak{M}(\mathbf{v}, \mathbf{w})=\mu^{-1}(0) / /\left(G_{\mathbf{v}}, \chi_{0}\right) \cong \mu_{\alpha}^{-1}(0) / /(\mathrm{G}(\alpha), \chi)
$$

in the notation of [9], where $\chi_{0}: G_{\mathbf{v}} \rightarrow K^{*}$ is the character defined by $\chi_{0}(g)=$ $\prod_{k \in I} \operatorname{det}\left(g_{k}^{-1}\right)$, and $\chi$ is the corresponding character of $\mathrm{G}(\alpha)$. This is a smooth variety. We say that a representation of $\Pi^{0}$ of dimension $\beta$ is $v$-cogenerated (where $v$ is a vertex with $\beta_{v}=1$ ) if it has no nonzero subrepresentation which is zero at $v$. This is dual to the notion of ' $v$-generated' of [4, Section 2]. By [18, Lemma 3.8] the points of $\mathfrak{M}(\mathbf{v}, \mathbf{w})$ are in one-to-one correspondence with isomorphism classes of $\infty$-cogenerated representations of $\Pi^{0}$ of dimension $\alpha$.

Now assume that $K$ is the field $\mathbb{C}$ of complex numbers. It is claimed in [18, Theorem 6.2] that $\mathfrak{M}(\mathbf{v}, \mathbf{w})$ is connected, but this is retracted in [19, Section 7.5]. Nakajima has mentioned to the author that connectivity can be recovered in some cases, the following argument works in all cases, however. 
Define $\lambda$ by $\lambda_{k}=-1$ for $k \in I$ and $\lambda_{\infty}=\sum_{k \in I} \mathbf{v}_{k}$. Thus $\lambda \cdot \alpha=0$, but $\lambda \cdot \beta \neq 0$ for all $0<\beta<\alpha$. If $\alpha$ is a root then trivially $\alpha \in \Sigma_{\lambda}$, so Theorem 1.2 implies that $\mu_{\alpha}^{-1}(\lambda) / / \mathrm{G}(\alpha)$ is nonempty and irreducible. On the other hand, if $\alpha$ is not a root, then Theorem 1.2 implies that there is no representation of $\Pi^{\lambda}$ of dimension $\alpha$, so that $\mu_{\alpha}^{-1}(\lambda) / / \mathrm{G}(\alpha)$ is empty. Now there is a bijection

$$
\mu_{\alpha}^{-1}(\lambda) / / \mathrm{G}(\alpha) \rightarrow \mu_{\alpha}^{-1}(0) / /(\mathrm{G}(\alpha), \chi) \cong \mathfrak{M}(\mathbf{v}, \mathbf{w}) .
$$

which is continuous for the analytic topology. (See [16, §§3,4] and [4, §3].) It follows that $\mathfrak{M}(\mathbf{v}, \mathbf{w})$ is either non-empty connected or empty, according to whether $\alpha$ is a root for $Q$ or not.

\section{Notation and Reflection Functors}

Let $Q$ be a quiver with vertex set $I$ and let $K$ be an algebraically closed field. In this section we introduce some standard notation, recall the reflection functors, and determine the effect of reflection functors on the fibres $\mu_{\alpha}^{-1}(\lambda)$.

We call elements of $\mathbb{Z}^{I}$ (or sometimes $\mathbb{R}^{I}$ ) vectors, and write $\varepsilon_{i}$ for the coordinate vector at a vertex $i$. We partially order $\mathbb{Z}^{I}$ via $\alpha \geqslant \beta$ if $\alpha_{i} \geqslant \beta_{i}$ for all $i$, and we write $\alpha>\beta$ to mean that $\alpha \geqslant \beta$ and $\alpha \neq \beta$. We say that $\alpha$ is sincere if $\alpha_{i}>0$ for all $i$.

The Ringel form on $\mathbb{Z}^{I}$ is defined by

$$
\langle\alpha, \beta\rangle=\sum_{i \in I} \alpha_{i} \beta_{i}-\sum_{a \in Q} \alpha_{t(a)} \beta_{h(a)} .
$$

Let $(\alpha, \beta)=\langle\alpha, \beta\rangle+\langle\beta, \alpha\rangle$ be its symmetrization. The corresponding quadratic form $q(\alpha)=\langle\alpha, \alpha\rangle=\frac{1}{2}(\alpha, \alpha)$ is the Tits form, and we have $p(\alpha)=1-q(\alpha)$. The fundamental region is the set of $0 \neq \alpha \in \mathbb{N}^{I}$ with connected support and with $\left(\alpha, \varepsilon_{i}\right) \leqslant 0$ for every vertex $i$.

If $i$ is a loopfree vertex (so $q\left(\varepsilon_{i}\right)=1$ ), there is a reflection $s_{i}: \mathbb{Z}^{I} \rightarrow \mathbb{Z}^{I}$ defined by $s_{i}(\alpha)=\alpha-\left(\alpha, \varepsilon_{i}\right) \varepsilon_{i}$. The real roots (respectively imaginary roots) are the elements of $\mathbb{Z}^{I}$ which can be obtained from the coordinate vector at a loopfree vertex (respectively \pm an element of the fundamental region) by applying some sequence of reflections at loopfree vertices.

There is a reflection $r_{i}: K^{I} \rightarrow K^{I}$ which is dual to $s_{i}$. It is defined by $r_{i}(\lambda)_{j}=$ $\lambda_{j}-\left(\varepsilon_{i}, \varepsilon_{j}\right) \lambda_{i}$. It satisfies $r_{i}(\lambda) \cdot \alpha=\lambda \cdot s_{i}(\alpha)$ for all $\alpha$.

We say that the reflection at a loopfree vertex $i$ is admissible for the pair $(\lambda, \alpha)$ if $\lambda_{i} \neq 0$. Let $\sim$ be the smallest equivalence relation on $K^{I} \times \mathbb{Z}^{I}$ with $(\lambda, \alpha) \sim\left(r_{i}(\lambda)\right.$, $\left.s_{i}(\alpha)\right)$ whenever the reflection at $i$ is admissible for $(\lambda, \alpha)$.

If the reflection at $i$ is admissible for $(\lambda, \alpha)$ then by $[5, \S 5]$ there is a reflection functor from representations of $\Pi^{\lambda}$ to representations of $\Pi^{r_{i}(\lambda)}$ which acts as as $s_{i}$ on dimension vectors. (In fact these reflection functors were discovered earlier, by Rump [21].)

We briefly describe the construction. Assume for simplicity that no arrow in $Q$ has tail at $i$, and let $H=\{a \in Q \mid h(a)=i\}$. Suppose that $V$ is a representation of $\Pi^{\lambda}$, 
given by vector spaces $V_{j}$ for each vertex $j$ and linear maps $V_{a}: V_{t(a)} \rightarrow V_{h(a)}$ for each arrow $a \in \bar{Q}$. Define $V_{\oplus}=\bigoplus_{a \in H} V_{t(a)}$ and let $\mu_{a}: V_{t(a)} \rightarrow V_{\oplus}$ and $\pi_{a}: V_{\oplus} \rightarrow V_{t(a)}$ be the canonical inclusions and projections. Define $\mu$ : $V_{i} \rightarrow V_{\oplus}$ and $\pi: V_{\oplus} \rightarrow V_{i}$ by

$$
\mu=\sum_{a \in H} \mu_{a} V_{a^{*}}, \quad \pi=\frac{1}{\lambda_{i}} \sum_{a \in H} V_{a} \pi_{a}
$$

The relations for $\Pi^{\lambda}$ ensure that $\pi \mu=1_{V_{i}}$, so that $\mu \pi$ is an idempotent endomorphism of $V_{\oplus}$. By definition the reflection functor sends $V$ to the representation $V^{\prime}$ of $\Pi^{r_{i}(\lambda)}$ given by vector spaces $V_{j}^{\prime}=V_{j}$ for $j \neq i$ and $V_{i}^{\prime}=\operatorname{Im}(1-\mu \pi)$, and by linear maps $V_{a}^{\prime}=V_{a}$ and $V_{a^{*}}^{\prime}=V_{a}^{\prime}$ for $a \in Q$ with $h(a) \neq i$, and

$$
V_{a}^{\prime}=-\lambda_{i}(1-\mu \pi) \mu_{a}: V_{t(a)}^{\prime} \rightarrow V_{i}^{\prime}, \quad V_{a^{*}}^{\prime}=\left.\pi_{a}\right|_{V_{i}^{\prime}}: V_{i}^{\prime} \rightarrow V_{t(a)}^{\prime},
$$

for $a \in H$.

We use the reflection functors to relate the schemes $\mu_{\alpha}^{-1}(\lambda)$ and $\mu_{s_{i}(\alpha)}^{-1}\left(r_{i}(\lambda)\right)$ (equipped with their scheme structure as fibres of the moment map). For our geometric arguments all schemes are quasiprojective over $K$, and all points are closed points.

LEMMA 2.1. If $0 \neq v \in K$ and $m, n$ are non-negative integers, then the projection from

$$
\begin{aligned}
\mathcal{S}= & \left\{\left(X, X^{*}, Y, Y^{*}\right) \mid X X^{*}=v 1, Y Y^{*}=-v 1, X^{*} X-Y^{*} Y=v 1\right\} \\
\subseteq & \operatorname{Mat}(n \times(n+m), K) \times \operatorname{Mat}((n+m) \times n, K) \times \\
& \times \operatorname{Mat}(m \times(n+m), K) \times \operatorname{Mat}((n+m) \times m, K)
\end{aligned}
$$

to

$$
\begin{aligned}
\mathcal{X} & =\left\{\left(X, X^{*}\right) \mid X X^{*}=v 1\right\} \\
& \subseteq \operatorname{Mat}(n \times(n+m), K) \times \operatorname{Mat}((n+m) \times n, K)
\end{aligned}
$$

is a principal $\mathrm{GL}(m, K)$-bundle. Moreover, the natural scheme structures on $\mathcal{S}$ and $\mathcal{X}$ given by the indicated relations are reduced.

Proof. By rescaling $X$ and $Y$ one can replace the equations by $X X^{*}=1, Y Y^{*}=1$, and $X^{*} X+Y^{*} Y=1$, so the matrices define inverse isomorphisms between $K^{n+m}$ and $K^{n} \oplus K^{m}$. The result is now standard.

LEMMA 2.2. Suppose given a pair $(\lambda, \alpha)$ with $\lambda \cdot \alpha=0$. If $i$ is a loopfree vertex with $\lambda_{i} \neq 0$ then there is a scheme $T$ and morphisms

$$
\mu_{\alpha}^{-1}(\lambda) \stackrel{f}{\leftarrow} T \stackrel{g}{\rightarrow} \mu_{s_{i}(\alpha)}^{-1}\left(r_{i}(\lambda)\right)
$$

where the map $f$ is a principal $\mathrm{GL}\left(s_{i}(\alpha)_{i}, K\right)$-bundle and $g$ is a principal $\mathrm{GL}\left(\alpha_{i}, K\right)$ bundle. In particular, $\mu_{\alpha}^{-1}(\lambda)$ and $\mu_{s_{i}(\alpha)}^{-1}\left(r_{i}(\lambda)\right)$ have the same number of irreducible 
components, and

$$
\operatorname{dim} \mu_{s_{i}(\alpha)}^{-1}\left(r_{i}(\lambda)\right)-s_{i}(\alpha) \cdot s_{i}(\alpha)=\operatorname{dim} \mu_{\alpha}^{-1}(\lambda)-\alpha \cdot \alpha .
$$

Proof. We suppose for simplicity that no arrow in $Q$ has tail at $i$. We can do this because the deformed preprojective algebra $\Pi^{\lambda}$ does not depend on the orientation of $Q$, see [5, Lemma 2.2]. (If $a$ were an arrow with tail at $i$ we could reverse it by sending $x_{a}$ to $x_{a^{*}}$ and $x_{a^{*}}$ to $-x_{a}$ for $x \in \operatorname{Rep}(\bar{Q}, \alpha)$.) Let $H=\{a \in Q \mid h(a)=i\}$.

Let $Q^{\prime}$ be the quiver obtained from $Q$ by deleting all arrows in $H$, and let $R^{\prime}=\operatorname{Rep}\left(\overline{Q^{\prime}}, \alpha\right)$. Letting $n=\alpha_{i}$ and

$$
m=s_{i}(\alpha)_{i}=-\alpha_{i}+\sum_{a \in H} \alpha_{t(a)}
$$

one can combine the matrices for the arrows incident at $i$ into block matrices, and identify

$$
\operatorname{Rep}(\bar{Q}, \alpha) \cong R^{\prime} \times \operatorname{Mat}(n \times(n+m), K) \times \operatorname{Mat}((n+m) \times n, K),
$$

so that if $x \in \operatorname{Rep}(\bar{Q}, \alpha)$ corresponds to a triple $\left(x^{\prime}, X, X^{*}\right)$ then

$$
\mu_{\alpha}(x)_{i}=\sum_{a \in H} x_{a} x_{a^{*}}=X X^{*}
$$

Also one can identify

$$
\operatorname{Rep}\left(\bar{Q}, s_{i}(\alpha)\right) \cong R^{\prime} \times \operatorname{Mat}(m \times(n+m), K) \times \operatorname{Mat}((n+m) \times m, K)
$$

and if $y$ corresponds to $\left(x^{\prime}, Y, Y^{*}\right)$ then

$$
\mu_{s_{i}(\alpha)}(y)_{i}=\sum_{a \in H} y_{a} y_{a^{*}}=Y Y^{*} .
$$

We now apply Lemma 2.1 with $v=\lambda_{i}$ to obtain a principal $\operatorname{GL}(m, K)$-bundle

$$
f^{\prime}: R^{\prime} \times \mathcal{S} \rightarrow R^{\prime} \times \mathcal{X} \cong\left\{x \in \operatorname{Rep}(\bar{Q}, \alpha) \mid \mu_{\alpha}(x)_{i}=\lambda_{i} 1\right\},
$$

where $\mathcal{S}$ and $\mathcal{X}$ are as in Lemma 2.1. Exchanging the role of the $X$ 's and $Y$ 's, we also obtain a principal $\mathrm{GL}(n, K)$-bundle

$$
g^{\prime}: R^{\prime} \times \mathcal{S} \rightarrow\left\{y \in \operatorname{Rep}\left(\bar{Q}, s_{i}(\alpha)\right) \mid \mu_{s_{i}(\alpha)}(y)_{i}=r_{i}(\lambda)_{i} 1\right\} .
$$

To show that $f^{\prime}$ and $g^{\prime}$ restrict to give a scheme $T$ and principal bundles $f$ and $g$, we need to show that for each vertex $j \neq i$ and each $z \in R^{\prime} \times \mathcal{S}$ we have

$$
\mu_{\alpha}\left(f^{\prime}(z)\right)_{j}-\lambda_{j} 1=\mu_{s_{i}(\alpha)}\left(g^{\prime}(z)\right)_{j}-r_{i}(\lambda)_{j} 1
$$

in $\operatorname{Mat}\left(\alpha_{j}, K\right)$.

Now if $x=f^{\prime}(z)$ and $y=g^{\prime}(z)$ then the relation $X^{*} X-Y^{*} Y=\lambda_{i} 1$ for $\mathcal{S}$ implies that $x_{a^{*}} x_{a}-y_{a^{*}} y_{a}=\lambda_{i} 1$ for any $a \in H$. Also $x_{a}=y_{a}$ for any arrow $a$ not incident at $i$, so that $x_{a^{*}} x_{a}-y_{a^{*}} y_{a}=0$ if $a \in Q$ and $h(a) \neq i$. Thus, if $j$ is a vertex different 
from $i$, we have

$$
\sum_{\substack{a \in Q \\ t(a)=j}} x_{a^{*}} x_{a}=\sum_{\substack{a \in Q \\ t(a)=j}} y_{a^{*}} y_{a}+N \lambda_{i} 1,
$$

where $N$ is the number of arrows from $j$ to $i$. Clearly we also have

$$
\sum_{\substack{a \in Q \\ h(a)=j}} x_{a} x_{a^{*}}=\sum_{\substack{a \in Q \\ h(a)=j}} y_{a} y_{a^{*}}
$$

since $j \neq i$. It follows that

$$
\mu_{\alpha}(x)_{j}-\mu_{s_{i}(\alpha)}(y)_{j}=-N \lambda_{i} 1=\left(\lambda_{j}-r_{i}(\lambda)_{j}\right) 1,
$$

as required.

\section{Lifting Representations from $Q$ to $\Pi^{\lambda}$}

Let $Q$ be a quiver with vertex set $I$ and let $\lambda \in K^{I}$. In this section we determine which representations of $Q$ lift to representations of $\Pi^{\lambda}$. That is, for $\alpha \in \mathbb{N}^{I}$ we determine the image of the projection $\pi: \mu_{\alpha}^{-1}(\lambda) \rightarrow \operatorname{Rep}(Q, \alpha)$. (For Dynkin quivers this problem has been studied by Rump [21]. His methods are, however, quite different.) In addition, if $U$ is a constructible subset of $\operatorname{Im}(\pi)$ which is $\mathrm{G}(\alpha)$-stable (that is, a union of $\mathrm{G}(\alpha)$-orbits), we relate the dimension of $\pi^{-1}(U)$ to the number of parameters of $\mathrm{G}(\alpha)$ on $U$. Recall that if $X$ is a scheme, $G$ is an algebraic group acting on $X$, and $U$ is a constructible subset of $X$ which is $G$-stable, then the number of parameters (or modularity) of $G$ on $U$, is defined by

$$
\operatorname{dim}_{G} U=\max _{d}\left(\operatorname{dim}\left(U \cap X_{d}\right)+d-\operatorname{dim} G\right)
$$

where $X_{d}$ is the locally closed subset of $X$ consisting of those points whose stabilizer has dimension $d$, so which have orbit of dimension $\operatorname{dim} G-d$.

LEMMA 3.1. If $x=\left(x_{a}\right)_{a \in Q} \in \operatorname{Rep}(Q, \alpha)$, then there is an exact sequence

$$
0 \rightarrow \operatorname{Ext}^{1}(x, x)^{*} \rightarrow \operatorname{Rep}\left(Q^{o p}, \alpha\right) \stackrel{c}{\rightarrow} \operatorname{End}(\alpha) \stackrel{t}{\rightarrow} \operatorname{End}(x)^{*} \rightarrow 0
$$

where c sends $\left(y_{a^{*}}\right) \in \operatorname{Rep}\left(Q^{o p}, \alpha\right)$ to $\sum_{a \in Q}\left[x_{a}, y_{a^{*}}\right]$ and $t$ sends $\left(\theta_{i}\right)$ to the linear map $\operatorname{End}(x) \rightarrow K$ sending $\left(\phi_{i}\right)$ to $\sum_{i} \operatorname{tr}\left(\theta_{i} \phi_{i}\right)$.

Proof. This is just a fuller statement of [5, Lemma 4.2].

LEMMA 3.2. If $\lambda \in K^{I}$ and $x$ is a representation of $Q$ which lifts to $\Pi^{\lambda}$, then $\sum_{i} \lambda_{i} \operatorname{tr}\left(\theta_{i}\right)=0$ for any $\theta \in \operatorname{End}(x)$.

Proof. Applying Lemma 3.1, since $x$ lifts, one deduces that $\lambda$ is in the image of $c$, so in the kernel of $t$. 
THEOREM 3.3. If $\lambda \in K^{I}$ then a representation of $Q$ lifts to a representation of $\Pi^{\lambda}$ if and only if the dimension vector $\beta$ of any direct summand satisfies $\lambda \cdot \beta=0$. Moreover, if $x \in \operatorname{Rep}(Q, \alpha)$ does lift, then $\pi^{-1}(x) \cong \operatorname{Ext}^{1}(x, x)^{*}$

Proof. If the representation lifts, and there is a direct summand of dimension $\beta$ then letting $\theta$ be the projection onto this summand, we have $\lambda \cdot \beta=0$ by Lemma 3.2.

For the converse, it suffices to prove the liftability of any indecomposable $x$ whose dimension vector $\alpha$ satisfies $\lambda \cdot \alpha=0$. Now any endomorphism $\theta$ of $x$ is the sum of a nilpotent matrix and a scalar matrix, so $\sum_{i} \lambda_{i} \operatorname{tr}\left(\theta_{i}\right)=0$. Thus, considering $\lambda$ as an element of $\operatorname{End}(\alpha)$, it is in the kernel of the map $t$ of Lemma 3.1. Thus $\lambda$ is in the image of $c$, and this gives a lift to $\Pi^{\lambda}$.

LEMMA 3.4 If $U$ is a $\mathrm{G}(\alpha)$-stable constructible subset of $\operatorname{Rep}(Q, \alpha)$ contained in the image of $\pi$, then

$$
\operatorname{dim} \pi^{-1}(U)=\operatorname{dim}_{\mathrm{G}(\alpha)} U+\alpha \cdot \alpha-q(\alpha) .
$$

If in addition $U$ is a $\mathrm{G}(\alpha)$-orbit, then $\pi^{-1}(U)$ is irreducible of dimension $\alpha \cdot \alpha-q(\alpha)$.

Proof. By partitioning $U$ we may suppose that all representations $x \in U$ have endomorphism ring of dimension $e$. Now if $x \in U$ then by Theorem 3.3 the fibre $\pi^{-1}(x)$ is isomorphic to $\operatorname{Ext}^{1}(x, x)^{*}$, so has dimension $e-q(\alpha)$ by Lemma 3.1. Thus $\operatorname{dim} \pi^{-1}(U)=\operatorname{dim} U+e-q(\alpha)$ On the other hand, each orbit of $\mathrm{G}(\alpha)$ on $U$ has dimension $\operatorname{dim} \mathrm{G}(\alpha)+1-e$, so $\operatorname{dim}_{\mathrm{G}(\alpha)} U=\operatorname{dim} U-1+e-\operatorname{dim} \mathrm{G}(\alpha)$. The dimension formula follows.

Now suppose in addition that $U=\mathrm{G}(\alpha) x$. Since $\operatorname{dim}_{\mathrm{G}(\alpha)} U=0$ the inverse image $\pi^{-1}(U)$ has dimension $\alpha \cdot \alpha-q(\alpha)$. It remains to prove that it is irreducible. Observe that $\mathrm{G}(\alpha)$ acts on $\mu_{\alpha}^{-1}(\lambda)$ and $\pi$ is equivariant. Now if $\pi^{-1}(U)$ is not irreducible one can find nonempty disjoint $\mathrm{G}(\alpha)$-stable open subsets $Z_{1}, Z_{2}$. But $\pi\left(Z_{i}\right)=U$, so $\pi^{-1}(x) \cap Z_{i}(i=1,2)$ are non-empty disjoint open subsets of $\pi^{-1}(x)$, which is impossible since $\pi^{-1}(x)$ is irreducible.

\section{Application of Kac's Theorem}

Let $Q$ be a quiver with vertex set $I$. Kac's Theorem [7, 8] asserts that the dimension vectors of indecomposable representations of $Q$ are exactly the positive roots for $Q$. Moreover, if $\alpha$ is a positive real root then there is a unique indecomposable representation of dimension $\alpha$, while if $\alpha$ is a positive imaginary root then $\operatorname{dim}_{\mathrm{G}(\alpha)} I(\alpha)=p(\alpha)$ where $I(\alpha) \subseteq \operatorname{Rep}(Q, \alpha)$ is the set of indecomposable representations.

We need some properties of $\operatorname{dim}_{G}$ which are easy to prove using Chevalley's Theorems.

LEMMA 4.1. Let $X$ be a scheme on which an algebraic group $G$ acts. Suppose that $Z \subseteq Y \subseteq X$ are constructible subsets, with $Y$ being $G$-stable and $Z$ being $H$-stable, 
where $H$ is a closed subgroup of $G$. If $Y=G Z$ and the intersection of $Z$ with any $G$-orbit in $Y$ is a finite union of $H$-orbits, then $\operatorname{dim}_{H} Z=\operatorname{dim}_{G} Y$.

LEMMA 4.2. Suppose that algebraic groups $G_{i}$ act on schemes $X_{i}$. If $Y_{i} \subseteq X_{i}$ are $G_{i}$-stable constructible subsets, then setting $G=\prod_{i} G_{i}$ and $Y=\prod_{i} Y_{i}$, we have $\operatorname{dim}_{G} Y=\sum_{i} \operatorname{dim}_{G_{i}} Y_{i}$.

For arbitrary $\alpha$, suppose that $\alpha=\beta^{(1)}+\cdots+\beta^{(r)}$ is a decomposition of $\alpha$ as a sum of positive roots for $Q$, and let $I\left(\beta^{(1)}, \ldots, \beta^{(r)}\right)$ be the subset of $\operatorname{Rep}(Q, \alpha)$ consisting of the representations whose indecomposable summands have dimension $\beta^{(t)}$. Clearly this is a $G(\alpha)$-stable constructible set.

LEMMA 4.3. If $\alpha=\beta^{(1)}+\cdots+\beta^{(r)}$ with the $\beta^{(t)}$ positive roots, then

$$
\operatorname{dim}_{\mathrm{G}(\alpha)} I\left(\beta^{(1)}, \ldots, \beta^{(r)}\right)=\sum_{t=1}^{r} p\left(\beta^{(t)}\right)
$$

Proof. Let $R^{\prime}=\operatorname{Rep}\left(Q, \beta^{(1)}\right) \times \cdots \times \operatorname{Rep}\left(Q, \beta^{(r)}\right)$, and consider it as a subset of $\operatorname{Rep}(Q, \alpha)$ using block-diagonal matrices. Let $I^{\prime}$ be the constructible subset of $R^{\prime}$ consisting of the elements in which each representation of dimension $\beta^{(t)}$ is indecomposable. By the Krull-Schmidt Theorem, Lemma 4.1 applies to the subsets

$$
I^{\prime} \subseteq I\left(\beta^{(1)}, \ldots, \beta^{(r)}\right) \subseteq \operatorname{Rep}(Q, \alpha)
$$

with $H$ the subgroup of $\mathrm{G}(\alpha)$ corresponding to the product $\prod_{t} \mathrm{G}\left(\beta^{(t)}\right)$. Thus

$$
\operatorname{dim}_{\mathrm{G}(\alpha)} I\left(\beta^{(1)}, \ldots, \beta^{(r)}\right)=\operatorname{dim}_{H} I^{\prime}=\sum_{t} \operatorname{dim}_{\mathrm{G}\left(\beta^{(t)}\right)} I\left(\beta^{(t)}\right)
$$

by Lemma 4.2 , and this is $\sum_{t} p\left(\beta^{(t)}\right)$ by Kac's Theorem.

THEOREM 4.4. Given a pair $(\lambda, \alpha)$ with $\lambda \cdot \alpha=0$, we have

$$
\operatorname{dim} \mu_{\alpha}^{-1}(\lambda)=\alpha \cdot \alpha-q(\alpha)+m,
$$

where $m$ is the maximum value of $\sum_{t=1}^{r} p\left(\beta^{(t)}\right)$, where $r \geqslant 1$ and $\alpha=\beta^{(1)}+\cdots+\beta^{(r)}$ is a decomposition with each $\beta^{(t)}$ a positive root and $\lambda \cdot \beta^{(t)}=0$. In particular, $\mu_{\alpha}^{-1}(\lambda)$ is non-empty if and only if there is such a decomposition.

Proof. Let $\pi: \mu_{\alpha}^{-1}(\lambda) \rightarrow \operatorname{Rep}(Q, \alpha)$ be the projection. We decompose $\operatorname{Rep}(Q, \alpha)$ as a union of sets of the form $I\left(\beta^{(1)}, \ldots, \beta^{(r)}\right)$, and consider the inverse images $\pi^{-1}\left(I\left(\beta^{(1)}, \ldots, \beta^{(r)}\right)\right)$. If some $\beta^{(t)}$ has $\lambda \cdot \beta^{(t)} \neq 0$ then this inverse image is empty. Otherwise, by Lemmas 3.4 and 4.3 this inverse image has dimension $\sum_{t=1}^{r} p\left(\beta^{(t)}\right)+\alpha \cdot \alpha-q(\alpha)$. The result follows.

We now turn to the proof of Theorem 1.1. We use Kac's 'canonical decomposition'. (See [8, Section 1.18].) 
LEMMA 4.5. If $\alpha \in \mathbb{N}^{I}$ has canonical decomposition $\alpha=\beta^{(1)}+\cdots+\beta^{(r)}$ with $r \geqslant 2$, then $p(\alpha)<\sum_{t} p\left(\beta^{(t)}\right)$.

Proof. This holds since $\left(\beta^{(s)}, \beta^{(t)}\right) \geqslant 0$ for $s \neq t$ by [8, Proposition 1.20].

Proof of Theorem 1.1. Let $d=\alpha \cdot \alpha-1+2 p(\alpha)$, the relative dimension of $\mu_{\alpha}$.

$(1) \Longrightarrow(2)$ Since $\mu_{\alpha}$ is flat, its image $U$ is an open subset of $\operatorname{End}(\alpha)_{0}$. Now apply [6, Corollaire 6.1.4] to the map $\operatorname{Rep}(\bar{Q}, \alpha) \rightarrow U$. Clearly $0 \in U$, so $\mu_{\alpha}^{-1}(0)$ has dimension $d$.

(2) $\Longrightarrow$ (3) Follows from Theorem 4.4.

(3) $\Longrightarrow$ (4) If $p(\alpha)<\sum_{t} p\left(\beta^{(t)}\right)$ for some decomposition $\alpha=\beta^{(1)}+\cdots+\beta^{(r)}$, then Lemma 4.5 shows that the inequality remains true when we replace each $\beta^{(t)}$ by all the terms in its canonical decomposition. But now the terms are positive roots.

(4) $\Longrightarrow$ (1) By Lemma 4.5 the canonical decomposition of $\alpha$ can only have one term. It follows that $\alpha$ is a Schur root. (See [8, Section 1.18].) This means that there is a representation of $Q$ of dimension $\alpha$ whose endomorphism algebra is the base field $K$. If $x \in \operatorname{Rep}(Q, \alpha)$ is such a representation, then the map $c$ of Lemma 3.1 has 1-dimensional cokernel. Since $\operatorname{Im}(c)$ is clearly contained in $\operatorname{End}(\alpha)_{0}$, it follows that $\operatorname{Im}(c)=\operatorname{End}(\alpha)_{0}$. It follows that any element of $\operatorname{End}(\alpha)_{0}$ is the image under the moment map $\mu_{\alpha}: \operatorname{Rep}(\bar{Q}, \alpha) \rightarrow \operatorname{End}(\alpha)_{0}$ of an element of $\operatorname{Rep}(\bar{Q}, \alpha)$ whose restriction to $Q$ is equal to $x$. In particular the moment map is surjective. We consider its fibres $\mu_{\alpha}^{-1}(\phi)$ with $\phi \in \operatorname{End}(\alpha)_{0}$. Let $\tilde{\pi}: \mu_{\alpha}^{-1}(\phi) \rightarrow \operatorname{Rep}(Q, \alpha)$ be the projection. Now if $U$ is a constructible $\mathrm{G}(\alpha)$-stable subset of $\operatorname{Rep}(Q, \alpha)$ then

$$
\operatorname{dim} \tilde{\pi}^{-1}(U) \leqslant \operatorname{dim}_{\mathrm{G}(\alpha)} U+\alpha \cdot \alpha-q(\alpha) .
$$

by the same argument as Lemma 3.4. It follows by Lemma 4.3 and the hypothesis that $\mu_{\alpha}^{-1}(\phi)$ has dimension at most $d$. Clearly, in fact, it is equidimensional of dimension $d$. Now [6, Proposition 6.1.5] implies that $\mu_{\alpha}$ is flat.

\section{Properties of the Set $\Sigma_{\lambda}$}

Throughout this section, $Q$ is a quiver with vertex set $I$. We prove some combinatorial results about the set $\Sigma_{\lambda}$ which are needed later. In the course of this, we obtain another characterization of $\Sigma_{\lambda}$, Theorem 5.6.

We write $R_{\lambda}^{+}$for the set of positive roots $\alpha$ with $\lambda \cdot \alpha=0$. Thus $\Sigma_{\lambda}$ is the set of $\alpha \in R_{\lambda}^{+}$with the property that $p(\alpha)>\sum p\left(\beta^{(t)}\right)$ for any decomposition $\alpha=\beta^{(1)}+\cdots+\beta^{(r)}$ with $r \geqslant 2$ and all $\beta^{(t)} \in R_{\lambda}^{+}$. We write $\mathbb{N} R_{\lambda}^{+}$for the set of sums of elements of $R_{\lambda}^{+}$(including 0 ).

LEMMA 5.1. Given any pair $(\lambda, \alpha)$ with $\alpha \in \mathbb{N} R_{\lambda}^{+}$, if $i$ is a vertex with $\lambda_{i}=0$ and $\left(\alpha, \varepsilon_{i}\right)>0$, then $\alpha-\varepsilon_{i} \in \mathbb{N} R_{\lambda}^{+}$.

Proof. Since $\left(\alpha, \varepsilon_{i}\right)>0$ there cannot be a loop at $i$, and therefore there is a reflection at $i$, although it is not admissible. Now $\alpha$ is a sum of positive roots $\sum_{t=1}^{r} \gamma^{(t)}$. If any $\gamma^{(t)}$ 
is equal to $\varepsilon_{i}$ then we're done. Otherwise all $s_{i}\left(\gamma^{(t)}\right)$ are positive roots, so in $R_{\lambda}^{+}$. Thus $s_{i}(\alpha)=\alpha-\left(\alpha, \varepsilon_{i}\right) \varepsilon_{i} \in \mathbb{N} R_{\lambda}^{+}$. Now adding on a suitable number of copies of $\varepsilon_{i} \in R_{\lambda}^{+}$, it follows that $\alpha-\varepsilon_{i} \in \mathbb{N} R_{\lambda}^{+}$.

LEMMA 5.2. If $(\lambda, \alpha) \sim\left(\lambda^{\prime}, \alpha^{\prime}\right)$ then

(1) $\alpha \in R_{\lambda}^{+}$if and only if $\alpha^{\prime} \in R_{\lambda^{\prime}}^{+}$

(2) $\alpha \in \mathbb{N} R_{\lambda}^{+}$if and only if $\alpha^{\prime} \in \mathbb{N} R_{\lambda^{\prime}}^{+}$

(3) $\alpha \in \Sigma_{\lambda}$ if and only if $\alpha^{\prime} \in \Sigma_{\lambda^{\prime}}$.

Proof. It suffices to prove (1), for then the other parts follow. Consider the admissible reflection at a loopfree vertex $i$ with $\lambda_{i} \neq 0$. Now if $\alpha$ is a positive root, then so is $s_{i}(\alpha)$, except when $\alpha=\varepsilon_{i}$. However, this case cannot occur since $\lambda \cdot \varepsilon_{i} \neq 0$, so that $\varepsilon_{i} \notin R_{\lambda}^{+}$.

LEMMA 5.3. Given any pair $(\lambda, \alpha)$ with $\alpha \in \mathbb{N} R_{\lambda}^{+}$, there is an equivalent pair $\left(\lambda^{\prime}, \alpha^{\prime}\right)$ with the property that $\left(\alpha^{\prime}, \varepsilon_{i}\right) \leqslant 0$ whenever $\lambda_{i}^{\prime} \neq 0$.

Proof. Amongst all equivalent pairs, choose $\left(\lambda^{\prime}, \alpha^{\prime}\right)$ with $\alpha^{\prime}$ minimal. This is possible since Lemma 5.2(2) ensures that $\alpha^{\prime} \geqslant 0$. Now if $\lambda_{i}^{\prime} \neq 0$ and there is a loop at $i$ then $\left(\alpha^{\prime}, \varepsilon_{i}\right) \leqslant 0$ is automatic, while if $\lambda_{i}^{\prime} \neq 0$ and $i$ is loopfree then $\left(\alpha^{\prime}, \varepsilon_{i}\right) \leqslant 0$, for otherwise the pair $\left(r_{i}\left(\lambda^{\prime}\right), s_{i}\left(\alpha^{\prime}\right)\right)$ is smaller.

LEMMA 5.4. Suppose that $0 \neq \alpha \in \mathbb{N} R_{\lambda}^{+}$and $\left(\alpha, \varepsilon_{i}\right) \leqslant 0$ for all vertices $i$ with $\lambda_{i} \neq 0$. If $(\beta, \alpha-\beta) \leqslant-2$ whenever $\beta, \alpha-\beta$ are nonzero and in $\mathbb{N} R_{\lambda}^{+}$, then $\alpha$ is either a coordinate vector or in the fundamental region.

Proof. Suppose that $\alpha$ is not a coordinate vector. We have $\left(\alpha, \varepsilon_{i}\right) \leqslant 0$ for all $i$, for if $\left(\alpha, \varepsilon_{i}\right)>0$ then we must have $\lambda_{i}=0$. Now the inequality $\left(\alpha, \varepsilon_{i}\right)>0$ implies that $i$ is loopfree, so $\left(\varepsilon_{i}, \varepsilon_{i}\right)=2$. Thus

$$
\left(\alpha-\varepsilon_{i}, \varepsilon_{i}\right)=\left(\alpha, \varepsilon_{i}\right)-2>-2 .
$$

This contradicts the hypotheses, since $\alpha-\varepsilon_{i} \in \mathbb{N} R_{\lambda}^{+}$by Lemma 5.1.

Next, the support quiver of $\alpha$ is connected. By assumption $\alpha \in \mathbb{N} R_{\lambda}^{+}$, so we can write $\alpha=\sum_{t=1}^{r} \gamma^{(t)}$ with the $\gamma^{(t)} \in R_{\lambda}^{+}$. Now supposing that the support of $\alpha$ is a disjoint union $C \cup D$ with no arrows connecting $C$ to $D$, then each $\gamma^{(t)}$ has support contained in either $C$ or $D$. Letting $\beta$ be the sum of the $\gamma^{(t)}$ with support contained in $C$ gives $(\beta, \alpha-\beta)=0$, contrary to the assumption.

Thus $\alpha$ is in the fundamental region.

LEMMA 5.5. If $0 \neq \alpha \in \mathbb{N} R_{\lambda}^{+}$and $(\beta, \alpha-\beta) \leqslant-2$ whenever $\beta, \alpha-\beta$ are nonzero and in $\mathbb{N} R_{\lambda}^{+}$, then $\alpha \in R_{\lambda}^{+}$.

Proof. By Lemma 5.2 we may replace the pair $(\lambda, \alpha)$ by any equivalent pair. Thus by Lemma 5.3 we may suppose that $\left(\alpha, \varepsilon_{i}\right) \leqslant 0$ whenever $\lambda_{i} \neq 0$. Now by the previous lemma $\alpha$ is either a coordinate vector or in the fundamental region. Thus it is in $R_{\lambda}^{+}$.

We now have another description of the set $\Sigma_{\lambda}$. 
THEOREM 5.6. If $\alpha \in \mathbb{N}^{I}$ then $\alpha \in \Sigma_{\lambda}$ if and only if $0 \neq \alpha \in \mathbb{N} R_{\lambda}^{+}$and $(\beta, \alpha-\beta) \leqslant-2$ whenever $\beta, \alpha-\beta$ are nonzero and in $\mathbb{N} R_{\lambda}^{+}$.

Proof. Suppose first that $\alpha \in \Sigma_{\lambda}$. Clearly we have $0 \neq \alpha \in \mathbb{N} R_{\lambda}^{+}$. We prove that $(\beta, \alpha-\beta) \leqslant-2$ whenever $\beta, \alpha-\beta$ are nonzero and in $\mathbb{N} R_{\lambda}^{+}$. For a contradiction, suppose that $(\beta, \alpha-\beta) \geqslant-1$ with $\beta, \alpha-\beta$ nonzero and in $\mathbb{N} R_{\lambda}^{+}$. It follows that $p(\alpha) \leqslant p(\beta)+p(\alpha-\beta)$. This gives a decomposition of the form

$$
\alpha=\sum_{t=1}^{r} \beta^{(t)}, \quad 0 \neq \beta^{(t)} \in \mathbb{N} R_{\lambda}^{+}, \quad p(\alpha) \leqslant \sum_{t=1}^{r} p\left(\beta^{(t)}\right)
$$

with $r=2$. Choose a decomposition of this type with $r$ maximal. Now each term $\beta^{(t)}$ in this sum is nonzero, and belongs to $\mathbb{N} R_{\lambda}^{+}$. By maximality, if $\gamma, \beta^{(t)}-\gamma$ are nonzero and in $\mathbb{N} R_{\lambda}^{+}$, then $p\left(\beta^{(t)}\right)>p(\gamma)+p\left(\beta^{(t)}-\gamma\right)$, so $\left(\gamma, \beta^{(t)}-\gamma\right)<-1$ and, hence, $\beta^{(t)} \in R_{\lambda}^{+}$by Lemma 5.5. Now this decomposition contradicts the fact that $\alpha \in \Sigma_{\lambda}$.

For the converse, suppose that $0 \neq \alpha \in \mathbb{N} R_{\lambda}^{+}$and $(\beta, \alpha-\beta) \leqslant-2$ whenever $\beta, \alpha-\beta$ are nonzero and in $\mathbb{N} R_{\lambda}^{+}$. By Lemma 5.5 we have $\alpha \in R_{\lambda}^{+}$. Assuming that $\alpha \notin \Sigma_{\lambda}$, there is a decomposition $\alpha=\sum_{t=1}^{r} \beta^{(t)}$ with $\beta^{(t)} \in R_{\lambda}^{+}$and with $p(\alpha) \leqslant$ $\sum_{t=1}^{r} p\left(\beta^{(t)}\right)$. It follows that $q(\alpha)-\sum_{t=1}^{r} q\left(\beta^{(t)}\right) \geqslant 1-r$, so

$$
\sum_{t=1}^{r}\left(\beta^{(t)}, \alpha-\beta^{(t)}\right)=\sum_{t \neq k}\left(\beta^{(t)}, \beta^{(k)}\right)=2\left(q(\alpha)-\sum_{t=1}^{r} q\left(\beta^{(t)}\right)\right) \geqslant 2-2 r .
$$

This implies that $\left(\beta^{(t)}, \alpha-\beta^{(t)}\right)>-2$ for some $t$, contrary to the assumption.

Note in particular that $\mathbb{N} R_{0}^{+}=\mathbb{N}^{I}$, giving the following simple description of $\Sigma_{0}$.

COROLLARY 5.7. If $\alpha \in \mathbb{N}^{I}$ then $\alpha \in \Sigma_{0}$ if and only if $\alpha>0$ and $(\beta, \alpha-\beta) \leqslant-2$ whenever $\beta \in \mathbb{N}^{I}$ and $0<\beta<\alpha$.

Combining Lemmas 5.2, 5.3, 5.4 and Theorem 5.6, we have proved:

THEOREM 5.8. If $\alpha \in \Sigma_{\lambda}$ then there is an equivalent pair $\left(\lambda^{\prime}, \alpha^{\prime}\right)$ with $\alpha^{\prime}$ either the coordinate vector at a loopfree vertex or in the fundamental region. The first case occurs if $\alpha$ is a real root; the second case if $\alpha$ is an imaginary root.

\section{Existence of Simple Representations}

Let $Q$ be a quiver with vertex set $I$. In this section we prove the implication (2) $\Longrightarrow(1)$ of Theorem 1.2.

LEMMA 6.1. If $X$ is an equidimensional scheme, $Y$ is an irreducible scheme and $f: X \rightarrow Y$ is a dominant morphism with all fibres irreducible of constant dimension $d$, then $X$ is irreducible. 
Proof. If $X$ is not irreducible, one can find disjoint irreducible open subsets $Z, Z^{\prime}$. Now the restriction of $f$ to $Z$ is a map $Z \rightarrow \overline{f(Z)}$ whose fibres have dimension at most $d$, so $d+\operatorname{dim} \overline{f(Z)} \geqslant \operatorname{dim} Z=\operatorname{dim} X=d+\operatorname{dim} Y$, so $\overline{f(Z)}=Y$, and for the general point $y \in Y$ the fibre $Z \cap f^{-1}(y)$ has dimension $d$. Similarly, for the general point $y \in Y$ the fibre $Z^{\prime} \cap f^{-1}(y)$ has dimension $d$. But $f^{-1}(y)$ is irreducible of dimension $d$, so these two sets must intersect. A contradiction since $Z, Z^{\prime}$ are disjoint.

Recall that a representation is said to be a brick if its endomorphism algebra is the base field $K$. We denote by $B(\alpha) \subseteq \operatorname{Rep}(Q, \alpha)$ the set of bricks for $Q$ of dimension $\alpha$.

If $\alpha$ is a dimension vector in the fundamental region and $q(\alpha)<0$ then by Kac's Lemma 1 (see [8, Section 1.10]), the set $B(\alpha)$ is a dense open subset of $\operatorname{Rep}(Q, \alpha)$, we have $\operatorname{dim}_{\mathrm{G}(\alpha)} B(\alpha)=p(\alpha)$, and $\operatorname{dim}_{\mathrm{G}(\alpha)}(I(\alpha) \backslash B(\alpha))<p(\alpha)$.

On the other hand, if $\alpha$ is in the fundamental region but $q(\alpha)=0$, then there need not be any bricks. In this case the support quiver of $\alpha$ is extended Dynkin, $\alpha$ is a multiple of the minimal imaginary root $\delta$, and we have the following result.

LEMMA 6.2. If $Q$ is an extended Dynkin quiver with minimal imaginary root $\delta$ and $\alpha=m \delta$ with $m \geqslant 1$, then every indecomposable representation of $Q$ of dimension $\alpha$ has endomorphism algebra of dimension $m$, and $I(\alpha)$ is an irreducible locally closed subset of $\operatorname{Rep}(Q, \alpha)$ with $\operatorname{dim}_{\mathrm{G}(\alpha)} I(\alpha)=1$.

Proof. Of course the fact that $\operatorname{dim}_{\mathrm{G}(\alpha)} I(\alpha)=1$ is one of the things that needs to be verified during the proof of Kac's Theorem.

The indecomposable representations of $Q$ of dimension $\alpha$ are known by the representation theory of extended Dynkin quivers. They all belong to the tubular family $T$ of $[20, \S 3.6(5),(6)]$. Recall from $[20, \S 3.1]$ that $T$ is a serial abelian category. Its simple objects are called simple regular modules.

We claim that an indecomposable in $T$, say in a tube of rank $r$, has dimension $\alpha$ if and only if it has a composition series in $T$ of length $m r$. Namely, suppose $Q$ has no oriented cycles. (The case of an oriented cycle follows by [20, §3.6 (6)].) Inspecting the proof of [20, Theorem 3.4], we see that the tube contains a module $W_{0}(\rho)$ of length $r$ and with a composition series involving each simple regular module in the tube. By the proof of $[20, \S 3.6(5)]$, the module $W_{0}$ corresponds to the maximal root for the corresponding Dynkin quiver, so $W_{0}(\rho)$ has dimension $\delta$. The claim follows.

It follows from this description that all indecomposables of dimension $\alpha$ have endomorphism algebra of dimension $m$. Now $I(\alpha)$ is locally closed by $[10, \S 2.5$ Proposition]. It is a union of infinitely many $G(\alpha)$-orbits. We show that each orbit is contained in an irreducible open subset of $I(\alpha)$ whose complement is a finite union of orbits. This implies the irreducibility of $I(\alpha)$.

If $U$ is a finite set of simple regular modules, the perpendicular category is the full subcategory

$$
U^{\perp}=\left\{M \mid \operatorname{Hom}(S, M)=\operatorname{Ext}^{1}(S, M)=0 \text { for all } S \in U\right\} .
$$


of the category of $K Q$-modules. Using the fact that the tubes are standard, and the Auslander-Reiten formula $[20, \S 2.4(5)]$ we see that an indecomposable of dimension $\alpha$ is in $U^{\perp}$ if and only if its regular socle (in $T$ ) is not in $U$.

We consider the orbit corresponding to an be an indecomposable module $X$ of dimension $\alpha$. Choose a finite collection $U$ of simple regular modules with the properties that (a) $U$ does not contain the regular socle of $X$; (b) at most one simple regular module in each tube is not in $U$; (c) if $Q$ has no oriented cycles then there is a unique tube which has all its simple regular modules in $U$, if $Q$ is an oriented cycle then no tube has all its simple regular modules in $U$. As in [3, Lemma 11.1], there is a homomorphism $\theta: K Q \rightarrow \operatorname{Mat}\left(N, K[x]\right.$ ) (where $N=\sum_{i} \delta_{i}$ ) such that restriction induces an equivalence from the category of $\operatorname{Mat}(N, K[x])$-modules to $U^{\perp}$. Thus there is a $K Q-K[x]$-bimodule $L$, free of rank $N$ over $K[x]$, such that the tensor product functor $L \otimes_{K[x]}$ - is an equivalence from $K[x]$-modules to $U^{\perp}$. It follows that as $\lambda \in K$ varies, the modules $L \otimes_{K[x]} K[x] /(x-\lambda)^{m}$ run through all indecomposables in $U^{\perp}$ of dimension $\alpha$. Choosing generators of $L$, this induces a morphism $\phi: K \rightarrow \operatorname{Rep}(Q, \alpha)$ from the affine line, whose image meets all $\mathrm{G}(\alpha)$-orbits in $I(\alpha)$ in $U^{\perp}$. Now consider the map

$$
\mathrm{G}(\alpha) \times K \rightarrow \operatorname{Rep}(Q, \alpha), \quad(g, \lambda) \mapsto g \phi(\lambda) .
$$

The image is contained in $I(\alpha)$, it contains the orbit for $X$, it is $\mathrm{G}(\alpha)$-stable, and it omits only finitely many orbits, so it is open in $I(\alpha)$. Since $\mathrm{G}(\alpha) \times K$ is irreducible, so is the image.

LEMMA 6.3. If $(\lambda, \alpha)$ is a pair with $\alpha \in \Sigma_{\lambda}$ then $\mu_{\alpha}^{-1}(\lambda)$ is irreducible of dimension $d=\alpha \cdot \alpha-1+2 p(\alpha)$. In particular it is a complete intersection.

Proof. By Theorem 5.8 and Lemma 2.2 we may reduce to the case where $\alpha$ is either a coordinate vector, or in the fundamental region. If $\alpha$ is a coordinate vector at a loopfree vertex, the result is trivial, so we suppose that $\alpha$ is in the fundamental region.

By Theorem 4.4 the space $\mu_{\alpha}^{-1}(\lambda)$ has dimension $d$. Moreover, since $d$ is the relative dimension of $\mu_{\alpha}$, it is equidimensional of dimension $d$. It remains to prove that it is irreducible.

Let $\pi$ be the projection $\mu_{\alpha}^{-1}(\lambda) \rightarrow \operatorname{Rep}(Q, \alpha)$. Thus the image of $\pi$ is given by Theorem 3.3, and any nonempty fibre $\pi^{-1}(x)$ is isomorphic to $\operatorname{Ext}^{1}(x, x)^{*}$, so is irreducible.

As in Theorem 4.4 we write $\mu_{\alpha}^{-1}(\lambda)$ as a union of sets of the form $\pi^{-1}\left(I\left(\beta^{(1)}, \ldots, \beta^{(r)}\right)\right)$. All except $\pi^{-1}(I(\alpha))$ have dimension strictly smaller than $d$.

Suppose first that $q(\alpha)<0$. As mentioned before Lemma 6.2, the set $B(\alpha)$ of bricks is a dense open subset of $\operatorname{Rep}(Q, \alpha)$. Now the set $\pi^{-1}(I(\alpha) \backslash B(\alpha))$ has dimension less than $d$. Thus it suffices to prove that $\pi^{-1}(B(\alpha))$ is irreducible. This space is open in $\mu_{\alpha}^{-1}(\lambda)$, so it is equidimensional of dimension $d$. Moreover every fibre of the map $\pi^{-1}(B(\alpha)) \rightarrow B(\alpha)$ is irreducible. Therefore $\pi^{-1}(B(\alpha))$ is irreducible by Lemma 6.1. 
If $q(\alpha)=0$ and $\alpha$ is indivisible the same argument holds. The set $B(\alpha)$ of bricks is a dense open subset, and there are only finitely many other orbits of indecomposables.

Finally suppose that $q(\alpha)=0$ and $\alpha$ is divisible. Thus the support of $\alpha$ is extended Dynkin with minimal positive imaginary root $\delta$, and $\alpha=m \delta$ for some $m \geqslant 2$. Now $\lambda \cdot \delta \neq 0$, for the decomposition $\alpha=\delta+\cdots+\delta$ contradicts the fact that $\alpha \in \Sigma_{\lambda}$. However $\lambda \cdot \alpha=0$, so the only possibility is that the field $K$ has characteristic $p>0$ and $m$ is a multiple of $p$. Now in fact $m=p$, for otherwise the decomposition $\alpha=p \delta+\cdots+p \delta$ contradicts the fact that $\alpha \in \Sigma_{\lambda}$.

Now the image of $\pi$ is contained in the set of representations of $Q$ with no summand of dimension $k \delta$ with $k<p$. Thus it consists of $I(\alpha)$ and only finitely many other orbits. Now $\pi^{-1}(I(\alpha))$ is obtained from $\mu_{\alpha}^{-1}(\lambda)$ by removing the inverse images of finitely many orbits. These inverse images have dimension strictly less than $d$. It follows that $\pi^{-1}(I(\alpha))$ is equidimensional of dimension $d$, and by the same argument $\pi^{-1}(I(\alpha))$ is irreducible, hence so is $\mu_{\alpha}^{-1}(\lambda)$.

LEMMA 6.4. Given a pair $(\lambda, \alpha)$ with $\lambda \cdot \alpha=0$, if $\beta \leqslant \alpha$ then the set of elements of $\mu_{\alpha}^{-1}(\lambda)$ such that the corresponding representation of $\Pi^{\lambda}$ has a subrepresentation of dimension vector $\beta$ is closed.

Proof. If $\operatorname{Gr}(k, n)$ denotes the Grassmannian of $k$-dimensional subspaces of an $n$-dimensional space, then the set of pairs consisting of an element of $\mu_{\alpha}^{-1}(\lambda)$ and a subrepresentation of dimension $\beta$ is a closed subset of $\mu_{\alpha}^{-1}(\lambda) \times \prod_{i \in I}\left(\beta_{i}, \alpha_{i}\right)$. Since Grassmannians are projective, its image under the projection onto $\mu_{\alpha}^{-1}(\lambda)$ is closed. (See [22, Lemma 3.1].)

LEMMA 6.5. Given a pair $(\lambda, \alpha)$ with $\lambda \cdot \alpha=0$, if $x \in \mu_{\alpha}^{-1}(\lambda)$ corresponds to a representation of $\Pi^{\lambda}$ which is a brick (that is, has endomorphism algebra equal to the base field $K$ ) then $\mu_{\alpha}^{-1}(\lambda)$ is smooth at $x$.

Proof. It suffices to prove that $\mu_{\alpha}$ is smooth at $x$. Now this holds by [3, Lemma 10.3]. Alternatively, note that $x$ corresponds to a brick if and only if $x$ has trivial stabilizer in $G(\alpha)$, and the claim is standard differential geometry.

LEMMA 6.6. Let $Q$ be an extended Dynkin quiver with minimal imaginary root $\delta$ and $\alpha=m \delta$ with $m \geqslant 1$. Let $\beta \in \mathbb{N}^{I}$. If the general element of $I(\alpha)$ has subrepresentations of dimension $\beta$ and $\alpha-\beta$, then $\beta$ is a multiple of $\delta$.

Proof. If $Q$ has no oriented cycles then clearly $\beta$ must have defect zero, so the subrepresentations of dimensions $\beta$ and $\alpha-\beta$ must be regular. Now the general element of $I(\alpha)$ is in a homogeneous tube, so all regular subrepresentations have dimension a multiple of $\delta$. If $Q$ is an oriented cycle then the same argument works, for the general element of $I(\alpha)$ involves $m$ copies of a simple representation of $Q$ of dimension $\delta$. 
THEOREM 6.7. If $(\lambda, \alpha)$ is a pair with $\alpha \in \Sigma_{\lambda}$ then $\mu_{\alpha}^{-1}(\lambda)$ is a reduced and irreducible complete intersection of dimension $\alpha \cdot \alpha-1+2 p(\alpha)$, and the general element of $\mu_{\alpha}^{-1}(\lambda)$ is a simple representation of $\Pi^{\lambda}$.

Proof. By Lemma 6.3, $\mu_{\alpha}^{-1}(\lambda)$ is irreducible of the right dimension. By Lemma 6.4, the simple representations are an open subset of $\mu_{\alpha}^{-1}(\lambda)$, so to show that the general element is simple it suffices to prove the existence of one simple representation of dimension $\alpha$. Now because the reflection functors of [5] are equivalences, we may assume as in Lemma 6.3 that $\alpha$ is a coordinate vector or in the fundamental region. Clearly there is a simple representation if $\alpha$ is a coordinate vector, so assume that $\alpha$ is in the fundamental region.

Assume for a contradiction that there is no simple representation. The irreducibility of $\mu_{\alpha}^{-1}(\lambda)$ implies that there is some $\beta$ such that the general representation of $\Pi^{\lambda}$ of dimension $\alpha$ has a subrepresentation of dimension $\beta$. Then by Lemma 6.4 this holds for every representation of $\Pi^{\lambda}$ of dimension $\alpha$.

First suppose that $q(\alpha)<0$ or $\alpha$ is indivisible. Then $\alpha$ is a Schur root. Thus the general representation of $Q$ of dimension $\alpha$ is indecomposable, so extends to a representation of $\Pi^{\lambda}$, and hence has a subrepresentation of dimension $\beta$. Similarly, the general representation of $Q^{o p}$ of dimension $\alpha$ has a subrepresentation of dimension $\beta$. Considering duals, this implies that the general representation of $Q$ of dimension $\alpha$ has a subrepresentation of dimension $\alpha-\beta$. Now by [22, Theorem 3.4] the general representation of dimension $\alpha$ decomposes as a direct sum of representations of dimension $\beta$ and $\alpha-\beta$, contrary to the fact that $\alpha$ is a Schur root.

Now suppose that $q(\alpha)=0$ and $\alpha$ is divisible. As in Lemma 6.3 the support of $\alpha$ is extended Dynkin with minimal positive imaginary root $\delta$ and $\alpha=p \delta$ where $K$ has characteristic $p>0$ and $\lambda \cdot \delta \neq 0$. Now any element of $I(\alpha)$ extends to a representation of $\Pi^{\lambda}$, and hence has a subrepresentation of dimension $\beta$. Similarly, by considering duals and the opposite quiver, any element of $I(\alpha)$ has a subrepresentation of dimension $\alpha-\beta$. Now by Lemma 6.6 we have $\beta=k \delta$ with $0<k<p$. But then $\lambda \cdot \beta \neq 0$, contradicting the fact that there are representations of $\Pi^{\lambda}$ of dimension $\beta$.

Finally, since the general element $x$ of $\mu_{\alpha}^{-1}(\lambda)$ is a simple representation, it is a brick, and hence by Lemma 6.5 it is a smooth point. Thus $\mu_{\alpha}^{-1}(\lambda)$ is generically reduced. Since it is also a complete intersection, hence Cohen-Macaulay, it is reduced.

\section{The Set $F_{\lambda}$}

In this section $Q$ is a quiver with vertex set $I$. If $\lambda \in K^{I}$, recall that $R_{\lambda}^{+}$is the set of positive roots $\alpha$ with $\lambda \cdot \alpha=0$. We define $F_{\lambda}$ to be the set of $\alpha \in R_{\lambda}^{+}$with the property that $\left(\alpha^{\prime}, \varepsilon_{i}\right) \leqslant 0$ for any $\left(\lambda^{\prime}, \alpha^{\prime}\right) \sim(\lambda, \alpha)$ and any vertex $i$ with $\lambda_{i}^{\prime}=0$. It is a sort of fundamental region with respect to $\lambda$. (Of course $F_{0}$ is precisely the fundamental region.) We prove that if there is a simple representation of $\Pi^{\lambda}$ of dimension $\alpha$, then 
either $(\lambda, \alpha)$ is equivalent to a pair $\left(\lambda^{\prime}, \alpha^{\prime}\right)$ with $\alpha^{\prime}$ the coordinate vector of a loopfree vertex, or $\alpha \in F_{\lambda}$.

By definition, if $(\lambda, \alpha) \sim\left(\lambda^{\prime}, \alpha^{\prime}\right)$ then $\alpha \in F_{\lambda}$ if and only if $\alpha^{\prime} \in F_{\lambda^{\prime}}$. Now Lemma 5.3 immediately implies the following result.

LEMMA 7.1. If $\alpha \in F_{\lambda}$ then there is an equivalent pair $\left(\lambda^{\prime}, \alpha^{\prime}\right) \sim(\lambda, \alpha)$ with $\alpha^{\prime}$ in the fundamental region. In particular $\alpha$ is an imaginary root.

LEMMA 7.2. If $\Pi^{\lambda}$ has a simple representation of dimension $\alpha$ and $i$ is a vertex, then either $\alpha=\varepsilon_{i}$, or $\lambda_{i} \neq 0$, or $\left(\alpha, \varepsilon_{i}\right) \leqslant 0$.

Proof. For simplicity we may suppose that no arrow has tail at $i$. Suppose that $\lambda_{i}=0$. Let $V$ be a simple representation of dimension vector $\alpha$, with vector space $V_{j}$ at each vertex $j$. Letting $V_{\oplus}=\oplus V_{t(a)}$, where the sum is over all arrows in $Q$ with head at $i$, the linear maps in $V$ combine to give maps

$$
V_{i} \underset{\phi}{\stackrel{\theta}{\rightleftarrows}} V_{\oplus}
$$

with $\phi \theta=0$.

Now if $\operatorname{Ker}(\theta) \neq 0$ then $V$ has a nonzero subrepresentation $W$ where $W_{i}=\operatorname{Ker}(\theta)$ and $W_{j}=0$ for all $j \neq i$.

On the other hand, if $\operatorname{Im}(\phi) \neq V_{i}$ then $V$ has a proper subrepresentation $W$ with $W_{i}=\operatorname{Im}(\phi)$ and $W_{j}=V_{j}$ for all $j \neq i$.

Thus, assuming that $V$ is simple and $\alpha \neq \varepsilon_{i}$, we deduce that $\theta$ is injective and $\phi$ is surjective. Since $\phi \theta=0$ the map $\phi$ induces a surjection $V_{\oplus} / \operatorname{Im}(\theta) \rightarrow V_{i}$, and hence $\operatorname{dim} V_{\oplus} \geqslant 2 \operatorname{dim} V_{i}$. Thus $\left(\alpha, \varepsilon_{i}\right)=2 \operatorname{dim} V_{i}-\operatorname{dim} V_{\oplus} \leqslant 0$.

LEMMA 7.3. If there is a simple representation of $\Pi^{\lambda}$ of dimension $\alpha$ then there is a pair $\left(\lambda^{\prime}, \alpha^{\prime}\right) \sim(\lambda, \alpha)$ with $\alpha^{\prime}$ a coordinate vector or in the fundamental region. In particular $\alpha$ is a root.

Proof. If $\left(\lambda^{\prime}, \alpha^{\prime}\right) \sim(\lambda, \alpha)$ then, because of the reflection functors, there is a simple representation of $\Pi^{\lambda^{\prime}}$ of dimension $\alpha^{\prime}$. In particular $\alpha^{\prime}>0$, so we can choose a pair $\left(\lambda^{\prime}, \alpha^{\prime}\right)$ with $\alpha^{\prime}$ minimal. Now either $\alpha^{\prime}$ is a coordinate vector, or in the fundamental region. Namely, supposing that $\alpha^{\prime}$ is not a coordinate vector, since there is a simple representation of dimension $\alpha^{\prime}$, it has connected support. Thus it suffices to prove that $\left(\alpha^{\prime}, \varepsilon_{i}\right) \leqslant 0$ for any vertex $i$. This is true if there is a loop at $i$, so we may suppose that $i$ is loopfree. If $\lambda_{i}=0$ then $\left(\alpha^{\prime}, \varepsilon_{i}\right) \leqslant 0$ by Lemma 7.2. If $\lambda_{i} \neq 0$ then the reflection at $i$ is admissible, and $\left(\alpha^{\prime}, \varepsilon_{i}\right) \leqslant 0$ by the minimality of $\alpha^{\prime}$.

LEMMA 7.4. If there is a simple representation of $\Pi^{\lambda}$ of dimension $\alpha$ then either $(\lambda, \alpha)$ is equivalent to a pair $\left(\lambda^{\prime}, \alpha^{\prime}\right)$ with $\alpha^{\prime}$ the coordinate vector of a loopfree vertex, or $\alpha \in F_{\lambda}$. 
Proof. Supposing that there is no equivalent pair $\left(\lambda^{\prime}, \alpha^{\prime}\right)$ with $\alpha^{\prime}$ the coordinate vector of a loopfree vertex, we show that $\alpha \in F_{\lambda}$. Of course $\alpha$ is a root by Lemma 7.3. If $\left(\lambda^{\prime}, \alpha^{\prime}\right) \sim(\lambda, \alpha)$ then there is a simple representation of $\Pi^{\lambda^{\prime}}$ of dimension $\alpha^{\prime}$. Now if $i$ is a vertex with $\lambda_{i}^{\prime}=0$ then either there is a loop at $i$, in which case $\left(\alpha^{\prime}, \varepsilon_{i}\right) \leqslant 0$ automatically, or if there is no loop at $i$, then $\left(\alpha^{\prime}, \varepsilon_{i}\right) \leqslant 0$ by Lemma 7.2.

\section{Classification of $\boldsymbol{F}_{\lambda} \backslash \Sigma_{\lambda}$}

Let $Q$ be a quiver with vertex set $I$. It follows from Theorem 6.7 and Lemma 7.4 that the set of imaginary roots in $\Sigma_{\lambda}$ is a subset of $F_{\lambda}$. In this section we show that this is quite close to being an equality. Not only is this a good way of determining the elements of $\Sigma_{\lambda}$ (especially when $\lambda=0$, so there are no admissible reflections), it is also essential for the proof of our characterization of the dimension vectors of simple representations of $\Pi^{\lambda}$.

THEOREM 8.1. If $(\lambda, \alpha)$ is a pair with $\alpha \in F_{\lambda} \backslash \Sigma_{\lambda}$, then after first passing to an equivalent pair, and then passing to the support quiver of $\alpha$ and the corresponding restrictions of $\lambda$ and $\alpha$, one of the following cases holds:

(I) $Q$ is extended Dynkin with minimal positive imaginary root $\delta$, and either $\lambda \cdot \delta=0$ and $\alpha=m \delta$ with $m \geqslant 2$ or, if the field $K$ has characteristic $p>0, \lambda \cdot \delta \neq 0$ and $\alpha=m^{\prime} p \delta$ with $m^{\prime} \geqslant 2$.

(II) I is a disjoint union $\mathcal{J} \cup \mathcal{K}$, with $\sum_{i \in \mathcal{K}} \lambda_{i} \alpha_{i}=0$, there is a unique arrow with one end in $\mathcal{J}$ and the other in $\mathcal{K}$, say connecting vertices $j \in \mathcal{J}$ and $k \in \mathcal{K}$, and $\alpha_{j}=\alpha_{k}=1$.

(III) $I$ is a disjoint union $\mathcal{J} \cup \mathcal{K}$, there is a unique arrow with one end in $\mathcal{J}$ and the other in $\mathcal{K}$, say connecting vertices $j \in \mathcal{J}$ and $k \in \mathcal{K}, \alpha_{j}=1$, the restriction of $Q$ to $\mathcal{K}$ is extended Dynkin with extending vertex $k$ and minimal positive imaginary root $\delta, \lambda \cdot \delta=0$, and the restriction of $\alpha$ to $\mathcal{K}$ is a multiple $m \delta$ with $m \geqslant 2$.

(Recall that if $Q$ is an extended Dynkin quiver and $\delta$ is its minimal imaginary root, then an extending vertex is a vertex $i$ with $\delta_{i}=1$.)

The proof of this theorem takes the rest of this section. Throughout, we assume that $\alpha \in F_{\lambda}$. In particular $\alpha$ is a root, so if it is sincere then $Q$ is connected. We say that $\beta \in \mathbb{N}^{I}$ is a $(-1)$-vector for the pair $(\lambda, \alpha)$ if $\beta, \alpha-\beta \in \mathbb{N} R_{\lambda}^{+}$and $(\beta, \alpha-\beta)=-1$. We say that $\beta$ is a divisor for $(\lambda, \alpha)$ if it is a $(-1)$-vector, $\left(\beta, \varepsilon_{i}\right) \leqslant 0$ for every vertex $i$, and $\left(\alpha-\beta, \varepsilon_{i}\right) \leqslant 0$ whenever $\left(\beta, \varepsilon_{i}\right)=0$. If $\beta$ is a divisor for $(\lambda, \alpha)$, then

$$
-1=(\beta, \alpha-\beta)=\sum_{i}(\alpha-\beta)_{i}\left(\beta, \varepsilon_{i}\right),
$$

and all terms in this sum are $\leqslant 0$. Thus there is a vertex $j$, which we call the critical vertex for $\beta$, with $\left(\beta, \varepsilon_{j}\right)=-1$ and $(\alpha-\beta)_{j}=1$, and for every other vertex $i$ one has $\left(\beta, \varepsilon_{i}\right)=0$ or $(\alpha-\beta)_{i}=0$. 
LEMMA 8.2. If $q(\alpha)<0$ then $(\beta, \alpha-\beta)<0$ for any $\beta$ with $\beta$ and $\alpha-\beta$ both nonzero and in $\mathbb{N} R_{\lambda}^{+}$.

Proof. Suppose that $(\beta, \alpha-\beta) \geqslant 0$ with $\beta, \alpha-\beta$ nonzero and in $\mathbb{N} R_{\lambda}^{+}$. By Lemma 7.1 there is an equivalent pair $\left(\lambda^{\prime}, \alpha^{\prime}\right)$ with $\alpha^{\prime}$ in the fundamental region. Applying the same sequence of reflections to $\beta$ gives a vector $\beta^{\prime}$ with $\left(\beta^{\prime}, \alpha^{\prime}-\beta^{\prime}\right) \geqslant 0$ and $\beta^{\prime}$ and $\alpha^{\prime}-\beta^{\prime}$ both nonzero. Now

$$
q\left(\alpha^{\prime}\right)=q\left(\alpha^{\prime}-\beta^{\prime}\right)+q\left(\beta^{\prime}\right)+\left(\beta^{\prime}, \alpha^{\prime}-\beta^{\prime}\right) \geqslant q\left(\alpha^{\prime}-\beta^{\prime}\right)+q\left(\beta^{\prime}\right)
$$

so by [10, Lemma 2, p123] the support quiver of $\alpha^{\prime}$ is extended Dynkin and $\alpha^{\prime}$ is a multiple of the minimal imaginary root. But this implies that $q(\alpha)=q\left(\alpha^{\prime}\right)=0$, a contradiction.

LEMMA 8.3. If $\alpha \in F_{\lambda} \backslash \Sigma_{\lambda}$ and $q(\alpha)<0$ then there is a (-1)-vector $\beta$ for $(\lambda, \alpha)$.

Proof. Combine the Lemma 8.2 with Theorem 5.6.

LEMMA 8.4. If $\alpha \in F_{\lambda} \backslash \Sigma_{\lambda}, q(\alpha)<0$ and $\beta$ is a (-1)-vector for $(\lambda, \alpha)$, then there is an equivalent pair $\left(\lambda^{\prime}, \alpha^{\prime}\right) \sim(\lambda, \alpha)$ which has a divisor $\beta^{\prime}$ satisfying $\beta^{\prime} \leqslant \beta$.

Proof. Amongst all $(-1)$-vectors $\beta^{\prime}$ for all pairs $\left(\lambda^{\prime}, \alpha^{\prime}\right)$ equivalent to $(\lambda, \alpha)$, choose $\beta^{\prime}$ to be minimal with $\beta^{\prime} \leqslant \beta$. Then choose an equivalent pair $\left(\lambda^{\prime}, \alpha^{\prime}\right)$ with $\alpha^{\prime}$ minimal amongst those having $\beta^{\prime}$ as a $(-1)$-vector.

We claim that $\left(\beta^{\prime}, \varepsilon_{i}\right) \leqslant 0$ for every vertex $i$. This is automatic if there is a loop at $i$, so we may suppose that $i$ is loopfree, and for a contradiction suppose that $\left(\beta^{\prime}, \varepsilon_{i}\right)>0$. We divide into two cases according to whether or not $\lambda_{i}^{\prime}=0$.

Suppose that $\lambda_{i}^{\prime} \neq 0$. This ensures that $\varepsilon_{i} \notin R_{\lambda^{\prime}}^{+}$, so any positive root in $R_{\lambda^{\prime}}^{+}$remains a positive root on applying the reflection $s_{i}$. Thus $s_{i}\left(\beta^{\prime}\right)$ and $s_{i}\left(\alpha^{\prime}-\beta^{\prime}\right)$ are in $\mathbb{N} R_{r_{i}}^{+}\left(\lambda^{\prime}\right)$ and, hence, $s_{i}\left(\beta^{\prime}\right)$ is a $(-1)$-vector for $\left(r_{i}\left(\lambda^{\prime}\right), s_{i}\left(\alpha^{\prime}\right)\right)$. Now since $\left(\beta^{\prime}, \varepsilon_{i}\right)>0$ it follows that $s_{i}\left(\beta^{\prime}\right)$ is strictly smaller than $\beta^{\prime}$, a contradiction.

Suppose on the other hand that $\lambda_{i}^{\prime}=0$. The vector $\beta^{\prime}-\varepsilon_{i}$ is in $\mathbb{N} R_{\lambda^{\prime}}^{+}$by Lemma 5.1. It is also nonzero, for if $\beta^{\prime}=\varepsilon_{i}$ then

$$
-1=\left(\varepsilon_{i}, \alpha^{\prime}-\varepsilon_{i}\right)=\left(\alpha^{\prime}, \varepsilon_{i}\right)-2,
$$

which is impossible since $\left(\alpha^{\prime}, \varepsilon_{i}\right) \leqslant 0$ because $\alpha \in F_{\lambda}$. Now $\beta^{\prime}-\varepsilon_{i}$ is a (-1)-vector for $\left(\lambda^{\prime}, \alpha^{\prime}\right)$, since

$$
\begin{aligned}
\left(\beta^{\prime}-\varepsilon_{i}, \alpha^{\prime}-\beta^{\prime}+\varepsilon_{i}\right) & =\left(\beta^{\prime}, \alpha^{\prime}-\beta^{\prime}\right)-\left(\varepsilon_{i}, \varepsilon_{i}\right)-\left(\alpha^{\prime}, \varepsilon_{i}\right)+2\left(\beta^{\prime}, \varepsilon_{i}\right) \\
& \geqslant-1-2-0+2=-1,
\end{aligned}
$$

so $\left(\beta^{\prime}-\varepsilon_{i}, \alpha^{\prime}-\beta^{\prime}+\varepsilon_{i}\right)=-1$ by Lemma 8.2 . This contradicts the minimality of $\beta^{\prime}$. Thus the claim is proved.

Finally, suppose that $i$ is a vertex with $\left(\beta^{\prime}, \varepsilon_{i}\right)=0$. If $\lambda_{i}^{\prime}=0$, then $\left(\alpha^{\prime}-\beta^{\prime}, \varepsilon_{i}\right)=$ $\left(\alpha^{\prime}, \varepsilon_{i}\right) \leqslant 0$ since $\alpha \in F_{\lambda}$. On the other hand, if $\lambda_{i}^{\prime} \neq 0$ then the reflection at $i$ is admissible for $\left(\lambda^{\prime}, \alpha^{\prime}\right)$, but $s_{i}$ has no effect on $\beta^{\prime}$, so if $\left(\alpha^{\prime}-\beta^{\prime}, \varepsilon_{i}\right)>0$ then $\beta^{\prime}$ is a $(-1)$-vector for $\left(r_{i}\left(\lambda^{\prime}\right),\left(s_{i} \alpha^{\prime}\right)\right)$, contradicting the minimality of $\alpha^{\prime}$. Thus $\beta^{\prime}$ is a divisor for $\left(\lambda^{\prime}, \alpha^{\prime}\right)$. 
LEMMA 8.5. Let $\beta$ be a divisor for $(\lambda, \alpha)$, and let $j$ be the critical vertex for $\beta$. Suppose that $\xi$ is a vector whose components are nonnegative real numbers, with support contained in the support of $\alpha-\beta$, with $\xi_{j}=0,\left(\xi, \varepsilon_{j}\right)=-1$ and with $\left(\xi, \varepsilon_{i}\right)$ nonnegative and integer-valued for every vertex $i \neq j$. Then there is at most one vertex $i$ at which $\left(\xi, \varepsilon_{i}\right)$ is strictly positive, and at this vertex $\left(\xi, \varepsilon_{i}\right)=1$.

Proof. If $i$ is a vertex with $\xi_{i} \neq 0$ then $i \neq j$ and by assumption $(\alpha-\beta)_{i} \neq 0$, so $\left(\beta, \varepsilon_{i}\right)=0$ and, hence, $\left(\alpha-\beta, \varepsilon_{i}\right) \leqslant 0$. It follows that $(\alpha-\beta, \xi) \leqslant 0$. Now since $(\alpha-\beta)_{j}=1$ we have

$$
(\alpha-\beta, \xi)=\sum_{i}(\alpha-\beta)_{i}\left(\xi, \varepsilon_{i}\right)=-1+\sum_{i \neq j}(\alpha-\beta)_{i}\left(\xi, \varepsilon_{i}\right)
$$

Now the terms in this last sum are non-negative integers, so at most one term is nonzero. Now if $\left(\xi, \varepsilon_{i}\right)>0$ then certainly $\xi_{i} \neq 0$, so by hypothesis $(\alpha-\beta)_{i} \neq 0$, and hence the corresponding term in the sum is nonzero.

LEMMA 8.6. Let $\beta$ be a divisor for $(\lambda, \alpha)$, and let $j$ be the critical vertex for $\beta$. Suppose there are vertices $v_{i}(1 \leqslant i \leqslant n)$ and the only arrows connected to the $v_{i}$ are of the following form (the orientation of the arrows is irrelevant): either

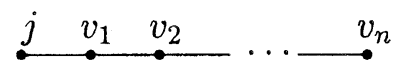

with $n \geqslant 1$, or

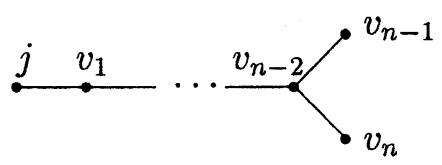

with $n \geqslant 3$. Then $(\alpha-\beta)_{v_{i}}=0$ for some $i$.

Proof. Supposing otherwise, we obtain a contradiction using Lemma 8.5. In the first case take $\xi$ to be the vector with $\xi_{v_{i}}=i$ for all $i$, and $\xi$ zero at all other vertices. In the second case take $\xi$ to be the vector with $\xi_{v_{i}}=i$ for $i \leqslant n-2$, $\xi_{v_{n-1}}=\xi_{v_{n}}=(n-1) / 2$, and $\xi$ zero at all other vertices.

LEMMA 8.7. Suppose that $\beta$ is a divisor for $(\lambda, \alpha)$, and let $j$ be the critical vertex for $\beta$. Let $Q^{\prime}$ be an extended Dynkin subquiver of $Q$ contained in the support of $\alpha-\beta$, and let $\delta$ be its minimal positive imaginary root. If for any vertex $i \in Q^{\prime}$ we define

$$
s_{i}=\sum_{\substack{a \in Q \\ h(a)=i}} \beta_{t(a)}+\sum_{\substack{a \in Q \backslash Q^{\prime} \\ t(a)=i}} \beta_{h(a)},
$$

then either $j \notin Q^{\prime}$ and $s_{i}=0$ for all vertices $i \in Q^{\prime}$, or $j \in Q^{\prime}$ and $\delta_{j}=\sum_{i \in Q^{\prime}} \delta_{i} s_{i}$.

Proof. For any vertex $i$ in $Q^{\prime}$ we have $\left(\beta, \varepsilon_{i}\right)=\left(\left.\beta\right|_{Q^{\prime}}, \varepsilon_{i}\right)_{Q^{\prime}}-s_{i}$. Thus $(\beta, \delta)=$ $-\sum_{i} \delta_{i} s_{i}$. Since $(\alpha-\beta)_{i} \neq 0$ for all $i$ in $Q^{\prime}$, we have $\left(\beta, \varepsilon_{i}\right)=0$ for any $i \neq j$. The result follows. 
LEMMA 8.8. If $Q^{\prime}$ is an extended Dynkin quiver and $r$ and $s$ are distinct extending vertices, then there is no vector $\gamma$ with integer components, with $\left(\gamma, \varepsilon_{r}\right)=1$, and with $\left(\gamma, \varepsilon_{i}\right)=0$ for all $i \neq r, s$.

Proof. Adding a suitable multiple of the minimal positive imaginary root $\delta$ we may assume that $\gamma_{r}=0$.

Suppose that $Q^{\prime}$ is of type $\tilde{A}_{n}$. Thus $Q^{\prime}$ has shape

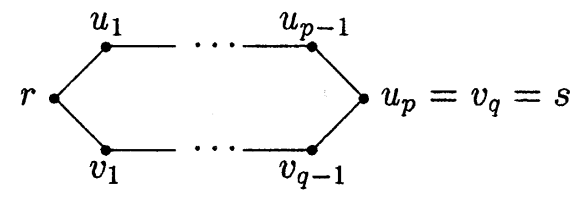

with $p, q \geqslant 1$. Now if $\gamma_{u_{1}}=x$ and $\gamma_{v_{1}}=y$ then the hypotheses imply that $x+y=-1$ and $\gamma_{u_{i}}=i x$ and $\gamma_{v_{i}}=i y$ for all $i$. Thus $p x=q y$, so $x$ and $y$ have the same sign. But then the equality $x+y=-1$ is impossible for $x, y$ integers.

Next suppose that $Q^{\prime}$ is of type $\tilde{D}_{n}$, in which case there are two possibilities for the location of $r$ and $s$. The first possibility is

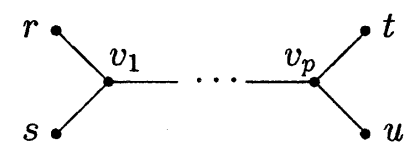

Now the hypotheses imply that $\gamma_{v_{1}}=-1$, but also $\gamma_{v_{p}}=2 \gamma_{t}=2 \gamma_{u}$, and then $\gamma_{v_{p}}=\gamma_{v_{p-1}}=\cdots=\gamma_{v_{1}}$, so $\gamma_{v_{1}}$ is even, a contradiction. The second possibility is

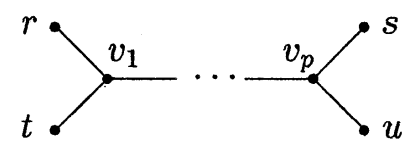

in which case $\gamma_{v_{1}}=-1$, but also $\gamma_{v_{1}}=2 \gamma_{t}$, a contradiction.

Finally, suppose that $Q^{\prime}$ is of type $\tilde{E}_{n}$. For type $\tilde{E}_{6}$, the components of $\gamma$ on the arm containing $r$ are successively $0,-1,-2$ (so -2 at the central vertex), but considering the arm not containing $r$ or $s$, if the component of $\gamma$ at the tip is $x$, then the components on the arm are $x, 2 x, 3 x$. Thus we need $3 x=-2$, which is impossible. For type $\tilde{E}_{7}$, the components of $\gamma$ on the arm containing $r$ are successively $0,-1,-2,-3$, but considering the shortest arm, if the component of $\gamma$ at the tip is $x$, then the component at the centre is $2 x$. Thus we need $2 x=-3$, which is impossible. Note that $\tilde{E}_{8}$ doesn't occur since it has only one extending vertex.

LEMMA 8.9. Suppose that $\beta$ is a divisor for $(\lambda, \alpha)$, that $j$ is the critical vertex for $\beta$, and that $\beta$ and $\alpha-\beta$ are both sincere. If $Q^{\prime}$ is an extended Dynkin subquiver of $Q$, then $j$ is contained in $Q^{\prime}$, and it is not an extending vertex for $Q^{\prime}$.

Proof. If $j$ is not in $Q^{\prime}$ then by Lemma 8.7 we have $s_{i}=0$ for all $i$. Since $\beta$ is sincere this implies that any arrow with one vertex in $Q^{\prime}$ is contained in $Q^{\prime}$. Since $Q$ is connected we must have $Q=Q^{\prime}$, but then $j$ is in $Q^{\prime}$, a contradiction. Thus $j$ is in $Q^{\prime}$. 
Now suppose that $j$ is an extending vertex for $Q^{\prime}$, that is, $\delta_{j}=1$, where $\delta$ is the minimal positive imaginary root for $Q^{\prime}$. Thus by Lemma 8.7 there is a unique arrow $a$ in $Q \backslash Q^{\prime}$ with one end in $Q^{\prime}$, say at vertex $\ell$. The other end cannot be in $Q^{\prime}$, say it is at vertex $k$. Then also $\beta_{k}=1$ and $\delta_{\ell}=1$. Now $\ell=j$, for otherwise by considering the restriction of $\beta$ to $Q^{\prime}$ we obtain a contradiction by Lemma 8.8.

Now $0=\left(\beta, \varepsilon_{k}\right) \leqslant 2 \beta_{k}-\beta_{j}-t=2-\beta_{j}-t$, where $t$ is the sum of all terms $\beta_{i}$ with $i$ a vertex not in $Q^{\prime}$ connected by an arrow to $k$. Thus $\beta_{j}$ is 1 or 2 .

If $\beta_{j}=2$ then $t=0$, so there are no arrows, apart from $a$ incident at $k$. Thus there is a linear quiver of length 1 attached to $j$, contrary to Lemma 8.6.

On the other hand, if $\beta_{j}=1$ then $t=1$, so $k$ must be connected to a unique vertex $u_{1}$ not in $Q^{\prime}$, and $\beta_{u_{1}}=1$. Now the condition $\left(\beta, \varepsilon_{u_{1}}\right)=0$ implies that $u_{1}$ must be connected to a unique vertex $u_{2} \neq k$ and $\beta_{u_{2}}=1$. Repeating in this way gives an infinite collection of distinct vertices $k, u_{1}, u_{2}, \cdots$. This is impossible.

Thus $j$ cannot be an extending vertex for $Q^{\prime}$.

LEMMA 8.10. If $\beta$ is a divisor for $(\lambda, \alpha)$ and $\beta$ and $\alpha-\beta$ are both sincere then $Q$ is a star with three arms.

Proof. Since every vertex of the extended Dynkin quiver of type $\tilde{A}_{n}$ is an extending vertex, by Lemma 8.9 the quiver $Q$ must be a tree.

Suppose that $Q^{\prime}$ is a subquiver of $Q$ which is extended Dynkin of type $\tilde{D}_{n}$, and let $\delta$ be the minimal positive imaginary root for $Q^{\prime}$. By Lemma 8.9,j must be contained in $Q^{\prime}$ and it is not an extending vertex. Thus $j$ is on the trunk of $Q^{\prime}$, and $\delta_{j}=2$. By Lemma 8.6, there must be arrows in $Q$ connecting to vertices on both sides of $j$, so by Lemma 8.7 there are two such arrows, they attach to extending vertices $k, \ell \in Q^{\prime}$, and we have $s_{k}=s_{\ell}=1$. Let $m$ be the vertex in $Q^{\prime}$ connected to $k$, and let $p$ be the other extending vertex in $Q^{\prime}$ connected to $m$ (or in case $Q^{\prime}$ is of type $\tilde{D}_{4}$, let $p$ be one of the other extending vertices with $p \neq \ell$ ). Since $\left(\beta, \varepsilon_{p}\right)=0$, we have $\beta_{m}=2 \beta_{p}$, so $\beta_{m}$ is even. On the other hand, since $\left(\beta, \varepsilon_{k}\right)=0$ we have $\beta_{m}+s_{k}=2 \beta_{k}$, so $\beta_{m}$ is odd, a contradiction.

Thus $Q$ contains no subquiver of type $\tilde{D}_{n}$, and so it is a star with three arms.

LEMMA 8.11. If $\beta$ is a divisor for $(\lambda, \alpha)$ then $\beta$ and $\alpha-\beta$ cannot both be sincere.

Proof. Supposing that $\beta$ and $\alpha-\beta$ are both sincere, we derive a contradiction. By Lemma 8.10, the quiver $Q$ is a star with three arms. Moreover, $j$ must be at the tip of one of the arms by Lemma 8.6. Note that $Q$ is not Dynkin or extended Dynkin since $(\beta, \beta)=\sum_{i} \beta_{i}\left(\beta, \varepsilon_{i}\right)=-\beta_{j}<0$. On deleting the vertex $j$, however, the quiver must be Dynkin by Lemma 8.9.

We say that $Q$ has type $(p, q, r)$ if the arm containing $j$ involves $p$ arrows and the other two arms involve $q$ and $r$ arrows respectively. Let $k$ and $\ell$ be the vertices at the tips of the second and third arms.

If $Q$ has type $(1, q, r)$, let $\xi$ be the vector which is 0 at $j$ and 1 at every other vertex. This gives a contradiction by Lemma 8.5 . 
If $Q$ has type $(2,1, r)$ for some $r$, let $\xi$ be the vector which is 0 at $j, 1$ at $k$ and the vertex adjacent to $j$, and 2 at all other vertices. This gives a contradiction by Lemma 8.5 .

If $Q$ has type (2, $q, r)$ with $q, r \geqslant 2$, then $j$ is an extending vertex for a subquiver of type $\widetilde{E}_{6}$. This is impossible by Lemma 8.9 .

Finally suppose that $Q$ has type $(p, q, r)$ with $p \geqslant 3$. Now $Q$ must contain an extended Dynkin subquiver $Q^{\prime}$. By Lemma 8.9, $Q^{\prime}$ must contain $j$, but the condition $p \geqslant 3$ forces $j$ to be an extending vertex for $Q^{\prime}$. This is impossible.

LEMMA 8.12. Suppose $\beta$ is a divisor for $(\lambda, \alpha)$. Assume $\alpha$ is sincere but $\beta$ is not. Then $\beta_{j}=0$, and decomposing $I$ as a disjoint union $\mathcal{J} \cup \mathcal{K}$ where $\mathcal{K}$ is the support of $\beta$ and $\mathcal{J}$ is the set of vertices where $\beta$ vanishes, there is a unique arrow connecting $\mathcal{J}$ to $\mathcal{K}$. It connects $j$ to some vertex $k \in \mathcal{K}$ with $\beta_{k}=1$.

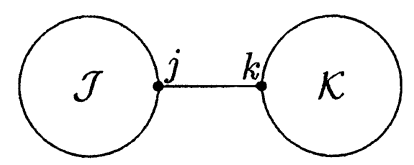

In addition there is a vertex $\ell \in \mathcal{K}$ (possibly equal to $k$ ) with the property that $\beta_{\ell}=1$, $\left(\alpha-\beta, \varepsilon_{\ell}\right)=-1$, and $\left(\alpha-\beta, \varepsilon_{i}\right)=0$ for all $i \in \mathcal{K}$ with $i \neq \ell$.

Proof. Since $Q$ is connected, at least one arrow $a$ connects $\mathcal{J}$ to $\mathcal{K}$. If its vertex in $\mathcal{J}$ is $i$, then clearly $\left(\beta, \varepsilon_{i}\right)<0$, so $i=j$. Now $\left(\beta, \varepsilon_{j}\right)=-1$, so there can be no other arrows between $j$ and $\mathcal{K}$, and if $k$ is the end of $a$ in $\mathcal{K}$, then $\beta_{k}=1$.

Observe that $\left(\alpha-\beta, \varepsilon_{i}\right) \leqslant 0$ for all $i \neq j$, since this is part of the definition of a divisor if $\left(\beta, \varepsilon_{i}\right)=0$, while if $\left(\beta, \varepsilon_{i}\right) \neq 0$ then we must have $(\alpha-\beta)_{i}=0$, and the assertion is clear. Now since $\beta_{j}=0$ we have

$$
-1=(\beta, \alpha-\beta)=\sum_{i \neq j} \beta_{i}\left(\alpha-\beta, \varepsilon_{i}\right)
$$

and in this sum all terms are $\leqslant 0$. Thus exactly one term is -1 , say corresponding to the vertex $i=\ell$, and all other terms are zero. The result follows.

LEMMA 8.13. If $Q^{\prime}$ is a Dynkin quiver it is not possible to find vertices $r$ and $s$ (possibly equal) and vectors $\beta$ and $\gamma$ with integer components, satisfying

$$
\begin{aligned}
& \beta_{r}=1, \quad\left(\beta, \varepsilon_{s}\right)=1, \quad\left(\beta, \varepsilon_{i}\right)=0 \text { for } i \neq s, \\
& \gamma_{s}=1, \quad\left(\gamma, \varepsilon_{r}\right)=1, \quad\left(\gamma, \varepsilon_{i}\right)=0 \text { for } i \neq r .
\end{aligned}
$$

Proof. First observe that $r \neq s$, for otherwise $(\beta, \beta)=1$, which is impossible since $(\beta, \beta)=2 q(\beta)$ is even.

Suppose there are vertices and arrows

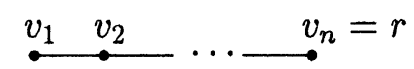


with $n \geqslant 2$, no other arrows attached to the $v_{i}(i<n)$, and all $v_{i} \neq s$. Then the conditions $\left(\beta, \varepsilon_{v_{i}}\right)=0$ imply by induction that $\beta_{v_{i}}=i \beta_{v_{1}}$. This is impossible since $\beta_{r}=1$. Similarly the configuration with $r$ and $s$ interchanged cannot occur. Thus one of three cases occurs. We eliminate each one in turn.

(1) $Q^{\prime}$ is of type $A_{n}$ and $r$ and $s$ are the opposite tips. Starting at the vertex $r$, the components of $\beta$ must be $1,2,3, \ldots, n$. But then $\left(\beta, \varepsilon_{s}\right)=2 n-(n-1)=$ $n+1 \neq 1$, a contradiction.

(2) $Q^{\prime}$ is a star with three arms, and $r$ and $s$ occur on the same arms, with one of them at the tip. Without loss of generality, assume that $s$ is at the tip. Letting $x=\beta_{s}$, working inwards from the vertex $s$ the components of $\beta$ must be $x, 2 x-1,3 x-2, \cdots$. Now if there are $p$ arrows between $r$ and $s$ we have $1=\beta_{r}=(p+1) x-p$, so $x=1$. Thus the component of $\beta$ at the centre of the star is also 1 , but considering either of the other arms, this is impossible.

(3) $Q^{\prime}$ is a star with three arms, and $r$ and $s$ occur as tips of different arms. Let the arm containing $r$ contain $p$ arrows, and let the arm containing $s$ contain $q$ arrows. Now, starting from $r$, the components of $\beta$ on the arm containing $r$ are $1,2, \ldots, p+1$. If $x=\beta_{s}$ then the components of $\beta$ on the arm containing $s$ are $x, 2 x-1,3 x-2, \ldots,(q+1) x-q$. Thus $p+1=(q+1) x-q$. Solving for $x$ this implies that $x \geqslant 2$ (since it is an integer), and then $p+1=q(x-1)+x>q+1$. Thus $p>q$. A similar argument with $r$ and $s$ interchanged gives $q>p$. Contradiction.

LEMMA 8.14. If $\beta$ is non-sincere divisor for $(\lambda, \alpha), \alpha$ is sincere and $\alpha-\beta$ is not sincere, then $(\lambda, \alpha)$ is of type (II).

Proof. If $\beta$ and $\alpha-\beta$ have disjoint support then it is easy to deduce from Lemma 8.12 that $(\lambda, \alpha)$ is of type (II). Thus, supposing that the supports of $\beta$ and $\alpha-\beta$ intersect, we need to derive a contradiction.

We decompose $\mathcal{K}$ as the disjoint union of $\mathcal{L}$, the set of vertices in $\mathcal{K}$ at which $\alpha-\beta$ vanishes, and $\mathcal{M}$, the intersection of $\mathcal{K}$ with the support of $\alpha-\beta$.

Since $Q$ is connected there is at least one arrow $b$ connecting $\mathcal{L}$ to $\mathcal{M}$. If its vertex in $\mathcal{L}$ is $i$, then clearly $\left(\alpha-\beta, \varepsilon_{i}\right)<0$, so $i$ is the vertex $\ell$ appearing in Lemma 8.12. Now $\left(\alpha-\beta, \varepsilon_{i}\right)=-1$ so there can be no other arrows between $\ell$ and $\mathcal{M}$, and if $m$ is the end of $b$ in $\mathcal{M}$ then $(\alpha-\beta)_{m}=1$. Thus $Q$ decomposes as follows (except that possibly $k=m$ ).

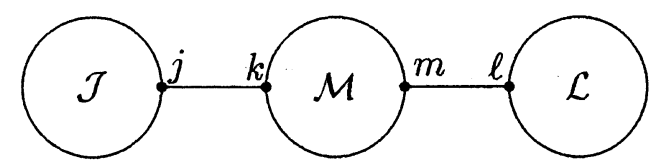

Let $Q^{\prime}$ be the restriction of $Q$ to $\mathcal{M}$. Now any subquiver of $Q^{\prime}$ must be connected by an arrow to a vertex at which $\beta$ is nonzero, so $Q^{\prime}$ cannot contain an extended Dynkin subquiver by Lemma 8.7. Thus $Q^{\prime}$ is Dynkin, and considering the restrictions of $\beta$ and $\alpha-\beta$ to $Q^{\prime}$, one gets a contradiction by Lemma 8.13 . 
LEMMA 8.15. If $\beta$ is non-sincere divisor for $(\lambda, \alpha)$ and $\alpha-\beta$ is sincere, then $(\lambda, \alpha)$ is of type (III).

Proof. Let $Q^{\prime}$ be the restriction of $Q$ to $\mathcal{K}$. Since $\alpha-\beta$ is sincere, we have $\left(\beta, \varepsilon_{i}\right)=0$ for all $i \neq j$, and hence $\left(\beta, \varepsilon_{i}\right)_{Q^{\prime}}=0$ for all $i \in Q^{\prime}$. Now $Q$ is connected, and hence so is $Q^{\prime}$, and then since $\beta$ has support $Q^{\prime}$, it follows from [10, Lemma 1, p123] that $Q^{\prime}$ is extended Dynkin and $\beta$ is a multiple of the minimal positive imaginary root $\delta$ for $Q^{\prime}$. Now $\beta_{k}=\beta_{\ell}=1$, so $\beta=\delta$ and $k$ and $\ell$ are extending vertices for $Q^{\prime}$. By Lemma 8.8 we have $k=\ell$. Let $\gamma$ be the restriction of $\alpha-\beta$ to $Q^{\prime}$. Then $\left(\gamma, \varepsilon_{i}\right)_{Q^{\prime}}=0$ for all $i \in Q^{\prime}$, so $\gamma$ is a multiple of $\delta$. The result follows.

Proof of Theorem 8.1. Suppose that $\alpha \in F_{\lambda} \backslash \Sigma_{\lambda}$. Since $\alpha$ is an imaginary root, $q(\alpha) \leqslant 0$. Suppose first that $q(\alpha)=0$. By passing to an equivalent pair, we may assume by Lemma 7.1 that $\alpha$ is in the fundamental region. Since $q(\alpha)=0$, this implies by [10, Lemma 1, p123] that the support of $\alpha$ is extended Dynkin and $\alpha$ is a multiple of the minimal imaginary root $\delta$, say $\alpha=m \delta$. If $\lambda \cdot \delta=0$ then clearly $m \geqslant 2$, for otherwise $\alpha \in \Sigma_{\lambda}$. On the other hand, if $\lambda \cdot \delta \neq 0$ then since $\lambda \cdot \alpha=0$ the field $K$ must have characteristic $p>0$ and $m$ is a multiple of $p$, say $m=m^{\prime} p$. Now $m^{\prime} \geqslant 2$ for otherwise $\alpha \in \Sigma_{\lambda}$. Thus we are in the situation of case (I).

Thus suppose that $q(\alpha)<0$. We replace $(\lambda, \alpha)$ by an equivalent pair to ensure that $\alpha$ has support as small as possible. Then we pass to the support quiver $Q^{\prime}$ of $\alpha$ and the restrictions $\left(\lambda^{\prime}, \alpha^{\prime}\right)$ of $\lambda$ and $\alpha$. Clearly $\alpha^{\prime} \in F_{\lambda^{\prime}} \backslash \Sigma_{\lambda^{\prime}}$. Observe that if we replace $\left(\lambda^{\prime}, \alpha^{\prime}\right)$ by any equivalent pair $\left(\lambda^{\prime \prime}, \alpha^{\prime \prime}\right)$, then $\alpha^{\prime \prime}$ is sincere (that is, has support $Q^{\prime}$ ), and $\left(\lambda^{\prime \prime}, \alpha^{\prime \prime}\right)$ can equally well be obtained from $(\lambda, \alpha)$ by applying the reflections first, and then passing to the support quiver.

Now by Lemma 8.3 there is a $(-1)$-vector $\beta$, for $\left(\lambda^{\prime}, \alpha^{\prime}\right)$, and hence a divisor $\beta^{\prime}$ for some equivalent pair $\left(\lambda^{\prime \prime}, \alpha^{\prime \prime}\right)$ by Lemma 8.4. Now $\beta^{\prime}$ and $\alpha^{\prime \prime}-\beta^{\prime}$ cannot both be sincere by Lemma 8.11 . Thus either $\beta^{\prime}$ is a non-sincere divisor, or we obtain a non-sincere divisor for some pair equivalent to $\left(\lambda^{\prime \prime}, \alpha^{\prime \prime}\right)$ on applying Lemma 8.4 to the $(-1)$-vector $\alpha^{\prime \prime}-\beta^{\prime}$ for $\left(\lambda^{\prime \prime}, \alpha^{\prime \prime}\right)$. Thus case (II) or (III) holds by Lemmas 8.14 and 8.15 .

\section{Nonexistence of Certain Simple Representations}

In this section we prove the following result. This is used in the next section to complete the proof of Theorem 1.2.

THEOREM 9.1. Let $Q^{\prime}$ be an extended Dynkin quiver, let $k$ be an extending vertex for $Q^{\prime}$, and let $Q$ be the quiver obtained from $Q^{\prime}$ by adjoining one vertex $j$ and one arrow $b: j \rightarrow k$. Let $I$ be the vertex set of $Q$, let $\delta \in K^{I}$ be the minimal positive imaginary root for $Q^{\prime}$, and let $\alpha=\varepsilon_{j}+m \delta$, where $m \geqslant 2$. If $\lambda \in K^{I}$ satisfies $\lambda_{j}=0$ and $\lambda \cdot \delta=0$, then there is no simple representation of $\Pi^{\lambda}$ of dimension vector $\alpha$.

Throughout this section we assume that $Q^{\prime}, Q, I, j, k, b, \delta, \alpha, m$ and $\lambda$ are as in the theorem. 
LEMMA 9.2. If $\alpha=\beta^{(1)}+\cdots+\beta^{(r)}$ with $\beta^{(t)} \in \mathbb{N}^{I} \backslash\{0\}$ for each $t$, then $\sum_{t=1}^{r} p\left(\beta^{(t)}\right) \leqslant p(\alpha)$, with equality exactly when all but one of the $\beta^{(t)}$ are equal to $\delta$.

Proof. Reordering, we may suppose that $\beta_{j}^{(1)}=1$ and $\beta_{j}^{(t)}=0$ for $t \neq 1$. Letting $\gamma=\beta^{(1)}-\varepsilon_{j}$, we have

$$
\sum_{t} p\left(\beta^{(t)}\right)=\gamma_{k}-q(\gamma)+\sum_{t \neq 1} p\left(\beta^{(t)}\right)
$$

Using the fact that the restriction of $q$ to $Q^{\prime}$ is positive semidefinite with radical $\mathbb{Z} \delta$, one can easily see that $p\left(\beta^{(t)}\right) \leqslant \beta_{k}^{(t)}$ for $t \neq 1$, with equality only possible if $\beta_{k}^{(t)}=0$ or $\beta^{(t)}=\delta$. Thus

$$
\sum_{t} p\left(\beta^{(t)}\right) \leqslant \alpha_{k}-q(\gamma)=m-q(\gamma) \leqslant m=p(\alpha)
$$

Now to have equality we must have $q(\gamma)=0$ and each $\beta^{(t)}(t \neq 1)$ either equal to $\delta$, or vanishing at $k$. But the condition $q(\gamma)=0$ implies that $\gamma$ is a multiple of $\delta$, and hence $\sum_{t \neq 1} \beta^{(t)}$ is also a multiple of $\delta$. This is impossible unless each of the terms is equal to $\delta$.

Let $\sigma: \operatorname{Rep}(Q, \alpha) \rightarrow \operatorname{Rep}\left(Q^{\prime}, m \delta\right)$ be the projection. If $U$ is a $\mathrm{G}(m \delta)$-stable subset of $\operatorname{Rep}\left(Q^{\prime}, m \delta\right)$, then clearly $\sigma^{-1}(U)$ is a $\mathrm{G}(\alpha)$-stable subset of $\operatorname{Rep}(Q, \alpha)$.

LEMMA 9.3. If $U$ is a non-empty open subset of $\operatorname{Rep}\left(Q^{\prime}, m \delta\right)$ which is $\mathrm{G}(m \delta)$-stable, then $\operatorname{dim}_{\mathrm{G}(\alpha)}\left(\operatorname{Rep}(Q, \alpha) \backslash \sigma^{-1}(U)\right)<p(\alpha)$.

Proof. For a dimension vector $\gamma$, we write $B(\gamma) \subseteq \operatorname{Rep}(Q, \gamma)$ for the set of bricks, and $I(\gamma)$ for the set of indecomposable representations. We claim that for $s \geqslant 0$ the vector $\gamma=\varepsilon_{j}+s \delta$ is a Schur root, and

$$
\operatorname{dim}_{\mathrm{G}(\gamma)}(I(\gamma) \backslash B(\gamma))<p(\gamma) .
$$

If $s=0$ this is trivial. If $s \geqslant 2$ then $\gamma$ is in the fundamental region, and the assertion follows from Kac $[8, \S 1.10$, Lemma 1]. Finally, if $s=1$ then $\gamma$ is obtained from the dimension vector $\delta$ by a reflection functor, see for example $[8, \S 1.7]$, and the assertion follows from the fact (which we also need later) that $\delta$ is a Schur root, and

$$
\operatorname{dim}_{\mathrm{G}(\delta)}(I(\delta) \backslash B(\delta))<p(\delta) .
$$

Indeed, $I(\delta) \backslash B(\delta)$ contains only finitely many orbits.

Now we decompose $\operatorname{Rep}(Q, \alpha)$ into sets $I\left(\beta^{(1)}, \ldots, \beta^{(r)}\right)$ as in Section 4 . We need to prove that

$$
\operatorname{dim}_{\mathrm{G}(\alpha)}\left(I\left(\beta^{(1)}, \ldots, \beta^{(r)}\right) \backslash \sigma^{-1}(U)\right)<p(\alpha) .
$$

By Lemmas 9.2 and 4.3 we only need to consider the sets $I\left(\varepsilon_{j}+s \delta, \delta, \ldots, \delta\right)$ for $0 \leqslant s \leqslant m$ (where there are $m-s$ copies of $\delta$ ). Now by the claim above and the argu- 
ment of Lemma 4.3 it suffices to prove that

$$
\operatorname{dim}_{\mathrm{G}(\alpha)}\left(B_{s} \backslash \sigma^{-1}(U)\right)<p(\alpha),
$$

where $B\left(\beta^{(1)}, \ldots, \beta^{(r)}\right)$ denotes the subset of $I\left(\varepsilon_{j}+s \delta, \delta, \ldots, \delta\right)$ in which the indecomposable summands are bricks.

Let $R_{s}^{\prime}=\operatorname{Rep}\left(Q, \varepsilon_{j}+s \delta\right) \times \operatorname{Rep}(Q, \delta) \times \cdots \times \operatorname{Rep}(Q, \delta)$, considered as a subset of $\operatorname{Rep}(Q, \alpha)$ using block-diagonal matrices. Let $B_{s}^{\prime}$ be the open subset of $R_{s}^{\prime}$ consisting of the elements in which each representation is a brick. Let $H$ the subgroup of $\mathrm{G}(\alpha)$ corresponding to the product $\mathrm{G}\left(\varepsilon_{j}+s \delta\right) \times \mathrm{G}(\delta) \times \cdots \times \mathrm{G}(\delta)$. By Lemma 4.1 we need to prove that $\operatorname{dim}_{H}\left(B_{s}^{\prime} \backslash \sigma^{-1}(U)\right)<p(\alpha)$. Since $H$ acts freely on $B_{s}^{\prime}$ this reduces to a question of dimension, and since $B_{s}^{\prime}$ is irreducible of dimension

$$
\begin{aligned}
& \operatorname{dim} \operatorname{Rep}\left(Q, \varepsilon_{j}+s \delta\right)+(m-s) \operatorname{dim} \operatorname{Rep}(Q, \delta) \\
& \quad=\operatorname{dim} H+p\left(\varepsilon_{j}+s \delta\right)+(m-s) p(\delta)=\operatorname{dim} H+p(\alpha),
\end{aligned}
$$

and $\sigma^{-1}(U)$ is an open subset, it suffices to prove that $B_{s}^{\prime}$ meets $\sigma^{-1}(U)$. In other words we need that $\sigma\left(B_{s}^{\prime}\right)$ meets $U$.

Now the canonical decomposition for dimension vector $m \delta$ is of the form $\delta+\cdots+\delta$, so $U$ contains a representation which is a direct sum of bricks of dimension $\delta$, and then since $U$ is $\mathrm{G}(m \delta)$-stable, it meets $P=\operatorname{Rep}\left(Q^{\prime}, s \delta\right) \times$ $\operatorname{Rep}\left(Q^{\prime}, \delta\right) \times \cdots \times \operatorname{Rep}\left(Q^{\prime}, \delta\right)$. Also the map $B_{s}^{\prime} \rightarrow P$ consists of an open inclusion followed by the projection, so the image $\sigma\left(B_{s}^{\prime}\right)$ is open in $P$. Since $P$ is irreducible, the two non-empty open subsets $\sigma\left(B_{s}^{\prime}\right)$ and $U \cap P$ must intersect. Thus $\sigma\left(B_{s}^{\prime}\right)$ meets $U$, as required.

Let $\pi: \mu_{\alpha}^{-1}(\lambda) \rightarrow \operatorname{Rep}(Q, \alpha)$ be the projection.

LEMMA 9.4. Under the map $\sigma \pi$, any irreducible component of $\mu_{\alpha}^{-1}(\lambda)$ dominates $\operatorname{Rep}\left(Q^{\prime}, m \delta\right)$.

Proof. Let $V$ be an irreducible component of $\mu_{\alpha}^{-1}(\lambda)$. Clearly $V$ is $\mathrm{G}(\alpha)$-stable, so $\sigma \pi(V)$ is $\mathrm{G}(m \delta)$-stable. Let $U$ be the complement of the closure of $\sigma \pi(V)$, and for a contradiction suppose that $U$ is non-empty. By Lemma 9.3 we have $\operatorname{dim}_{\mathrm{G}(\alpha)}\left(\operatorname{Rep}(Q, \alpha) \backslash \sigma^{-1}(U)\right)<p(\alpha)$. Now $\quad V \subseteq \pi^{-1}\left(\operatorname{Rep}(Q, \alpha) \backslash \sigma^{-1}(U)\right)$, so by Lemma 3.4 we have

$$
\operatorname{dim} V<p(\alpha)+\alpha \cdot \alpha-q(\alpha)=\operatorname{dim} \operatorname{Rep}(\bar{Q}, \alpha)-\operatorname{dim} \operatorname{End}(\alpha)_{0} .
$$

This is impossible since $\mu_{\alpha}^{-1}(\lambda)$ is a fibre of the moment map, so every irreducible component has dimension at least $\operatorname{dim} \operatorname{Rep}(\bar{Q}, \alpha)-\operatorname{dim} \operatorname{End}(\alpha)_{0}$.

Recall that a ring epimorphism $A \rightarrow B$ is said to be pseudoflat if $\operatorname{Tor}_{1}^{A}(B, B)=0$. This is relevant because of [3, Theorem 0.7]. 
LEMMA 9.5. If $N=\sum_{i} \delta_{i}$ then there is a pseudoflat epimorphism $\theta: K Q^{\prime} \rightarrow$ $\operatorname{Mat}(N, K[x])$ such that the general representation of $Q^{\prime}$ of dimension $m \delta$ is the restriction of a $\operatorname{Mat}(N, K[x])$-module,

Proof. This is standard. See [3, Lemma 11.1].

LEMMA 9.6. If $A \rightarrow B$ is a pseudoflat epimorphism of $K$-algebras, and $M$ is a left $A$-module, then the map

$$
\left(\begin{array}{cc}
A & M \\
0 & K
\end{array}\right) \rightarrow\left(\begin{array}{cc}
B & B \otimes_{A} M \\
0 & K
\end{array}\right)
$$

is a pseudoflat epimorphism.

Proof. By [1, Proposition 5.2] it suffices to observe that the diagram

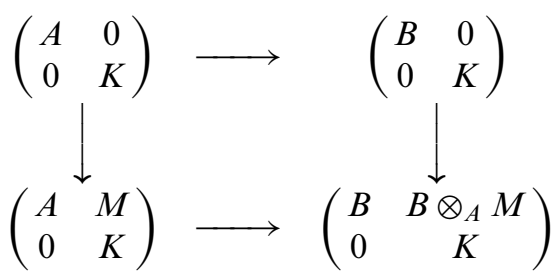

is a pushout in the category of rings.

LEMMA 9.7. Suppose that $f$ and $g$ are endomorphisms of a vector space $V$ of dimension $m \geqslant 2$. If the commutator $[f, g]$ has rank at most one, then $V$ has a nontrivial proper subspace invariant under $f$ and $g$.

Proof. Replacing $f$ by $f-\xi 1$ for some eigenvalue $\xi$ of $f$, we may suppose that $f$ is singular. Also we may suppose that $f \neq 0$, for otherwise one can take an invariant subspace for $g$. Let $v_{1}, \ldots, v_{r}$ be a basis of $\operatorname{Im}(f)$, and extend it to a basis $v_{1}, \ldots, v_{m}$ of $V$. Let $w_{1}, \ldots, w_{s}$ be a basis of $\operatorname{Ker}(f)$, and extend it to a basis $w_{1}, \ldots, w_{m}$ of $V$. With respect to these bases, we compute the matrices of $f$ and $g$. With the rows and columns indexed by the $v_{i}$, let $g$ take the block form $\left(\begin{array}{cc}X & Y \\ Z & W\end{array}\right)$, and with the rows and columns indexed by the $w_{i}$, let $g$ take the block form $\left(\begin{array}{ll}P & Q \\ R & S\end{array}\right)$. Now with the rows indexed by by the $v_{i}$ and the columns indexed by the $w_{i}$, the map $f$ takes block form $\left(\begin{array}{ll}0 & C \\ 0 & 0\end{array}\right)$ with $C$ invertible, and then $[f, g]$ takes the form

$$
\left(\begin{array}{cc}
C R & C S-X C \\
0 & -Z C
\end{array}\right)
$$

Now the rank one hypothesis implies that $C R=0$ or $Z C=0$, so that $R=0$ or $Z=0$. In the first case $\operatorname{Ker}(f)$ is an invariant subspace; in the second case $\operatorname{Im}(f)$ is invariant. 
Proof of Theorem 9.1. Choose a pseudoflat epimorphism $\theta$ as in Lemma 9.5. By Lemma 9.6 it induces a pseudoflat epimorphism

$$
\phi: K Q=\left(\begin{array}{cc}
K Q^{\prime} & K Q^{\prime} e_{k} \\
0 & K
\end{array}\right) \longrightarrow\left(\begin{array}{cc}
\operatorname{Mat}(N, K[x]) & \operatorname{Mat}(N, K[x]) \theta\left(e_{k}\right) \\
0 & K
\end{array}\right) .
$$

Denote the right hand algebra by $R$. Now the fact that $\delta_{k}=1$ implies that $\operatorname{Mat}(N, K[x]) \theta\left(e_{k}\right)$ is an indecomposable projective $\operatorname{Mat}(N, K[x])$-module. Thus $R$ is Morita equivalent to

$$
\left(\begin{array}{cc}
K[x] & K[x] \\
0 & K
\end{array}\right) \cong K Q^{\prime \prime}
$$

where $Q^{\prime \prime}$ is the quiver with two vertices $j, k$ and arrows $b: j \rightarrow k$ and $a: k \rightarrow k$.

Identify $\lambda \in K^{I}$ with the corresponding element of $K \otimes_{\mathbb{Z}} K_{0}(K Q)$, and then identify $\Pi^{\lambda}$ with the algebra $\Pi^{\lambda}(K Q)$ as in [3, Theorem 0.2]. Now $\phi$ induces a map $\phi_{\lambda}: \Pi^{\lambda} \rightarrow \Pi^{\phi_{*}(\lambda)}(R)$, and by [3, Theorem 0.7] the diagram

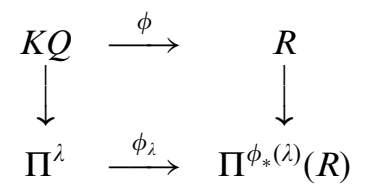

is a pushout in the category of rings.

Now suppose that there is a simple representation of $\Pi^{\lambda}$ of dimension vector $\alpha$. Since the simple representations form an open subset of $\mu_{\alpha}^{-1}(\lambda)$, it follows by Lemma 9.4 that the set of simple representations dominates $\operatorname{Rep}\left(Q^{\prime}, m \delta\right)$. Thus there is a simple representation $S$ whose restriction to $Q^{\prime}$ is the restriction by $\theta$ of a $\operatorname{Mat}(N, K[x])$-module. Thus the restriction of $S$ to $Q$ is the restriction by $\phi$ of an $R$-module. Now since the diagram above is a pushout, it follows that $S$ is naturally a $\Pi^{\phi_{*}(\lambda)}(R)$-module, and clearly it must be simple. We show that this is impossible.

By [3, Corollary 5.5] the ring $\Pi^{\phi_{*}(\lambda)}(R)$ is Morita equivalent to $\Pi^{\mu}\left(Q^{\prime \prime}\right)$, where $\mu_{j}=\mu_{k}=0$ by [3, Lemma 11.2]. Moreover $S$ corresponds to a simple representation $T$ of $\Pi^{\mu}\left(Q^{\prime \prime}\right)$ of dimension vector $\gamma$ with $\gamma_{j}=1$ and $\gamma_{k}=m$. Now the arrows $a$ and $a^{*}$ are endomorphisms of the vector space $T_{k}$ with commutator equal to $b^{*} b$. Since $\operatorname{dim} T_{j}=1$ it follows that this commutator has rank at most one, so by Lemma 9.7, $T_{k}$ has a non-trivial proper subspace invariant under $a$ and $a^{*}$. Now this subspace and its image under $b^{*}$ are a nontrivial proper subrepresentation of $T$. This is a contradiction.

\section{Dimension Vectors of Simple Representations}

Let $Q$ be a quiver with vertex set $I$. In this section we complete the proof of Theorem 1.2. All that remains is to prove the implication (1) $\Longrightarrow(2)$, that is, if $\alpha$ is the dimension vector of a simple representation of $\Pi^{\lambda}$ then $\alpha \in \Sigma_{\lambda}$. Thus suppose there is a simple representation of $\Pi^{\lambda}$ of dimension $\alpha$, and for a contradiction assume 
that $\alpha \notin \Sigma_{\lambda}$. Observe that there cannot be an equivalent pair $\left(\lambda^{\prime}, \alpha^{\prime}\right)$ with $\alpha^{\prime}$ a coordinate vector, for then clearly $\alpha^{\prime} \in \Sigma_{\lambda^{\prime}}$, a contradiction by Lemma 5.2. Thus by Lemma 7.4 we have $\alpha \in F_{\lambda}$, and so Theorem 8.1 applies. Thus we may assume that we are in a situation as in (I), (II) or (III), and to obtain a contradiction it suffices to show that in each case there is no simple representation.

Case (I). By [3] there is a Conze embedding

$$
\Pi^{\lambda} \rightarrow \operatorname{Mat}(N, K\langle x, y \mid x y-y x=\lambda \cdot \delta\rangle)
$$

where $N=\sum_{i} \delta_{i}$. If $\lambda \cdot \delta=0$ this embedding shows that $\Pi^{\lambda}$ satisfies the identities of $N \times N$ matrices, so any simple representation has dimension at most $N$. Thus $\Pi^{\lambda}$ cannot have a simple representation of dimension vector $m \delta$ with $m \geqslant 2$. If $\lambda \cdot \delta \neq 0$ and $K$ has characteristic $p>0$ then $K\langle x, y \mid x y-y x=\lambda \cdot \delta\rangle$ embeds in $\operatorname{Mat}\left(p, K\left[x^{p}, y\right]\right)$. Thus $\Pi^{\lambda}$ satisfies the identities of $p N \times p N$ matrices, so any simple representation has dimension at most $p N$. Thus $\Pi^{\lambda}$ cannot have a simple representation of dimension vector $m^{\prime} p \delta$ with $m^{\prime} \geqslant 2$.

Case (II). Since up to isomorphism $\Pi^{\lambda}$ does not depend on the orientation of $Q$, we may assume that the arrow connecting $j$ and $k$ is $b: j \rightarrow k$. Suppose that $V$ is a representation of $\Pi^{\lambda}$ of dimension $\alpha$. Let $V_{i}$ be the vector space corresponding to vertex $i$ and let $V_{a}$ be the linear map corresponding to an arrow $a$. Now at any vertex $i$ we have

$$
\sum_{h(a)=i} V_{a} V_{a^{*}}-\sum_{t(a)=i} V_{a^{*}} V_{a}=\lambda_{i} 1_{V_{i}}
$$

Taking traces and summing over all vertices $i \in \mathcal{K}$, almost all terms cancel, and one obtains $\operatorname{tr}\left(V_{b} V_{b^{*}}\right)=0$. Now since $\alpha_{j}=\alpha_{k}=1$ this implies that $V_{b}=0$ or $V_{b^{*}}=0$. In the first case $\oplus_{i \in \mathcal{J}} V_{i}$ is a subrepresentation of $V$; in the second case $\oplus_{i \in \mathcal{K}} V_{i}$ is a subrepresentation of $V$. Thus $V$ is not simple, as required.

Case (III). Suppose that $V$ is a representation of $\Pi^{\lambda}$ of dimension $\alpha$. As in case (II) we may assume that the arrow connecting $j$ and $k$ is $b: j \rightarrow k$, and furthermore $\operatorname{tr}\left(V_{b} V_{b^{*}}\right)=0$. Thus $\operatorname{tr}\left(V_{b^{*}} V_{b}\right)=0$, and since $\alpha_{j}=1$ this implies that $V_{b^{*}} V_{b}=0$.

Let $Q^{\prime \prime}$ be the quiver obtained from $Q$ by deleting all vertices in $\mathcal{J}$ except $j$, and all arrows with head and tail in $\mathcal{J}$. Let $\alpha^{\prime \prime}$ be the restriction of $\alpha$ to $\mathcal{K} \cup\{j\}$, and let $\lambda^{\prime \prime}$ be the vector with $\lambda_{j}^{\prime \prime}=0$ and $\lambda_{i}^{\prime \prime}=\lambda_{i}$ for $i \in \mathcal{K}$. In view of the observation above, the restriction $V^{\prime \prime}$ of $V$ to $\overline{Q^{\prime \prime}}$ is a representation of the deformed preprojective algebra $\Pi^{\lambda^{\prime \prime}}$ for the quiver $Q^{\prime \prime}$, of dimension vector $\alpha^{\prime \prime}$. Now by Theorem 9.1, the representation $V^{\prime \prime}$ cannot be simple, so it has a non-trivial proper subrepresentation $W$. Now $V_{j}$ is one-dimensional, so either $W_{j}=0$ or $W_{j}=V_{j}$. In the first case $W$ can be extended to a subrepresentation of $V$ by defining $W_{i}=0$ for all $i \in \mathcal{J} \backslash\{j\}$; in the second case $W$ can be extended to a subrepresentation of $V$ by defining $W_{i}=V_{i}$ for all $i \in \mathcal{J} \backslash\{j\}$. Thus $V$ is not simple, as required. 


\section{Quotient Schemes}

In this section $K$ is an algebraically closed field of characteristic zero, and $Q$ is a quiver with vertex set $I$.

Proof of Theorem 1.3. By [12, Theorem 2] the quotient scheme $\operatorname{Rep}(\bar{Q}, \alpha) / / \mathrm{G}(\alpha)$ is a disjoint union of locally closed strata according to the representation type of the semisimple representations. Now the quotient $\mu_{\alpha}^{-1}(\lambda) / / \mathrm{G}(\alpha)$ can be identified with a closed subset of $\operatorname{Rep}(\bar{Q}, \alpha) / / \mathrm{G}(\alpha)$, so the semisimple representations of a given type $\tau$ form a locally closed subset $S(\tau)$. Suppose that $\tau$ is the type

$$
\tau=\left(k_{1}, \beta^{(1)} ; \cdots ; k_{r}, \beta^{(r)}\right) .
$$

and consider the subset $Z$ of

$$
\mu_{\beta^{(1)}}^{-1}(\lambda) \times \cdots \times \mu_{\beta^{(r)}}^{-1}(\lambda)
$$

consisting of those tuples $\left(x_{1}, \ldots, x_{r}\right)$ with the $x_{t}$ corresponding to pairwise non-isomorphic simple representations. Clearly $Z$ is an open subset and $S(\tau)$ is the image of the map

$$
f: Z \rightarrow \mu_{\alpha}^{-1}(\lambda) / / \mathrm{G}(\alpha)
$$

sending $\left(x_{1}, \ldots, x_{r}\right)$ to the direct sum of the $x_{t}$ with multiplicities. Thus $S(\tau)$ is irreducible. Now the group $H=\mathrm{G}\left(\beta^{(1)}\right) \times \cdots \times \mathrm{G}\left(\beta^{(r)}\right)$ acts freely on $Z$, and any fibre of $f$ is a finite union of $H$-orbits. Thus

$$
\operatorname{dim} S(\tau)=\operatorname{dim} Z-\operatorname{dim} H=\sum_{t=1}^{r} 2 p\left(\beta^{(t)}\right),
$$

as required.

Proof of Corollary 1.4. Since $\mu_{\alpha}^{-1}(\lambda)$ is reduced and irreducible, so is the quotient $\mu_{\alpha}^{-1}(\lambda) / / \mathrm{G}(\alpha)$. Now the stratum of simple representations has dimension $2 p(\alpha)$, and all other strata have strictly smaller dimension.

Remark 11.1. If $(\lambda, \alpha)$ is a pair with $\lambda \cdot \alpha=0$ but $\lambda \cdot \beta \neq 0$ for all $0<\beta<\alpha$, then clearly $\alpha \in \Sigma_{\lambda}$ if and only if it is a positive root. If it is a positive root then every element of $\mu_{\alpha}^{-1}(\lambda)$ must be a simple representation of $\Pi^{\lambda}$ by [5, Lemma 4.1]. Thus $\mu_{\alpha}^{-1}(\lambda)$ is smooth by Lemma 6.5 , and the map

$$
\mu_{\alpha}^{-1}(\lambda) \rightarrow \mu_{\alpha}^{-1}(\lambda) / / \mathrm{G}(\alpha)
$$

is a principal étale fibre space for the group $\mathrm{G}(\alpha)$ by Luna's slice theorem [13, §III.1, Corollaire 1]. It follows in this case that $\mu_{\alpha}^{-1}(\lambda) / / \mathrm{G}(\alpha)$ is smooth. It would be interesting to know about the singularities of $\mu_{\alpha}^{-1}(\lambda)$ and $\mu_{\alpha}^{-1}(\lambda) / / \mathrm{G}(\alpha)$ for general $\lambda$ and $\alpha$. 


\section{Appendix. Application to Kac's Theorem}

In this appendix we show how the lifting results of Section 3 can be used to give a simple proof of part of Kac's Theorem assuming that the base field $K$ has characteristic zero. Recall [8, Section 1.10] that the proof of Kac's Theorem uses two key lemmas

KAC'S LEMMA 1. If $\alpha$ is in the fundamental region and $q(\alpha)<0$ then the set $B(\alpha)$ of bricks (representations with endomorphism algebra equal to $K$ ) is a dense open subset of $\operatorname{Rep}(Q, \alpha)\left(\right.$ so $\left.\operatorname{dim}_{G(\alpha)} B(\alpha)=p(\alpha)\right)$ and $\operatorname{dim}_{\mathrm{G}(\alpha)}(I(\alpha) \backslash B(\alpha))<p(\alpha)$.

KAC'S LEMMA 2. The number of indecomposable representations of dimension $\alpha$ (if it is finite) and $\operatorname{dim}_{\mathrm{G}(\alpha)} I(\alpha)$ are independent of the orientation of $Q$.

Kac's proof of Lemma 1 is quite natural and straightforward. On the other hand, his proof of Lemma 2 is roundabout, and involves reducing to finite fields and then using counting arguments. It would be nice to avoid Lemma 2, or find a direct proof of it.

PROPOSITION A.1. If $\alpha \in \mathbb{Z}^{I}$ then $\alpha$ is a positive root if and only if for the general element of $\left\{\lambda \in K^{I} \mid \lambda \cdot \alpha=0\right\}$ there is an indecomposable representation of $\Pi^{\lambda}$ of dimension $\alpha$.

Proof. Let $S(\alpha)$ be the statement that for the general element of $\left\{\lambda \in K^{I} \mid \lambda \cdot \alpha=0\right\}$ there is an indecomposable representation of $\Pi^{\lambda}$ of dimension $\alpha$.

Since $K$ has characteristic zero, if $i$ is a loopfree vertex and $\alpha$ is not a multiple of $\varepsilon_{i}$, then the general element of the set $\left\{\lambda \in K^{I} \mid \lambda \cdot \alpha=0\right\}$ has $\lambda_{i} \neq 0$. For such $\lambda$ there is a reflection functor relating representations of $\Pi^{\lambda}$ of dimension $\alpha$ and representations of $\Pi^{r_{i}(\lambda)}$ of dimension $s_{i}(\alpha)$. It follows that $S(\alpha)$ holds if and only if $S\left(s_{i}(\alpha)\right)$ holds.

Note also that if $i$ is a loopfree vertex and $\alpha$ is not a multiple of $\varepsilon_{i}$, then $\alpha$ is a positive root if and only if $s_{i}(\alpha)$ is a positive root.

Now, by applying a sequence of reflections to reduce $\alpha$, it suffices to prove the theorem in the following three cases.

(1) $\alpha$ is a multiple of the coordinate vector at a loopfree vertex, say $\alpha=k \varepsilon_{i}$. In this case $\alpha$ is a positive root if and only if $k=1$, and also clearly $S(\alpha)$ holds if and only if $k=1$.

(2) $\alpha$ is in the fundamental region. In this case $\alpha$ is a positive root. Also, by Kac's Lemma 1 and the theory of extended Dynkin quivers, there is an indecomposable representation of $Q$ of dimension $\alpha$, and by Theorem 3.3 this lifts to a representation of $\Pi^{\lambda}$ for any $\lambda$ with $\lambda \cdot \alpha=0$. Thus $S(\alpha)$ holds.

(3) $\alpha$ has disconnected support, or a strictly negative component. In this case $\alpha$ is not a positive root, and $S(\alpha)$ is false.

COROLLARY A.2. If there is an indecomposable representation of $Q$ of dimension $\alpha$ then $\alpha$ is a positive root. 
Proof. By Theorem 3.3 this representation lifts to an indecomposable representation of $\Pi^{\lambda}$ for any $\lambda$ with $\lambda \cdot \alpha=0$. Thus $S(\alpha)$ holds.

Remark A.3. Suppose that $K=\mathbb{C}$ and $Q$ has no oriented cycles. In this case Schofield [23] has used Euler characteristics to construct the positive part of the Kac-Moody Lie algebra associated to $Q$. In the course of his proof he shows that if $\alpha$ is a positive root then there is an indecomposable representation of $Q$ of dimension $\alpha$. This result and Corollary 1.2 give a proof of Kac's characterization which completely avoids finite fields.

If $\alpha$ is a positive real root, then the unique indecomposable representation of $Q$ of dimension $\alpha$ may be constructed as follows. Choose a sequence of reflections

$$
\varepsilon_{i}=\alpha^{(0)}, \alpha^{(1)}, \ldots, \alpha^{(m)}=\alpha
$$

with $i$ a loopfree vertex, $\alpha^{(t)}=s_{i_{t}}\left(\alpha^{(t-1)}\right)$ for $t \geqslant 1$, and $\alpha^{(t)}$ not a coordinate vector for $t \geqslant 1$. Let $\lambda^{(0)} \in K^{I}$ be the vector with $\lambda_{i}^{(0)}=0$ and $\lambda_{j}^{(0)}=1$ for all $j \neq i$, and define $\lambda^{(t)}=r_{i_{t}}\left(\lambda^{(t-1)}\right)$ for $t \geqslant 1$.

PROPOSITION A.4. With the hypotheses above, the reflection at $i_{t} i$ s admissible for $\left(\lambda^{(t)}, \alpha^{(t)}\right)$ for all $t$. Moreover, there is a unique indecomposable representation of $Q$ of dimension $\alpha$, and it may be obtained from the trivial representation of $\Pi^{\lambda^{(0)}}$ of dimension $\varepsilon_{i}$ by applying successively the reflection functors at the vertices $i_{t}$, and then restricting the resulting representation of $\Pi^{\lambda^{(m)}}$ to $Q$.

Proof. Since $K$ has characteristic zero, $\lambda^{(0)} \cdot \beta \neq 0$ for any $\operatorname{root} \beta$ which is not equal to $\pm \varepsilon_{i}$ (for some component $\beta_{j}$ with $j \neq i$ must be nonzero, and all components have the same sign). It follows that $\lambda^{(t)} \cdot \beta \neq 0$ for any root $\beta$ which is not equal to $\pm \alpha^{(t)}$. In particular $\lambda^{(t)} \cdot \varepsilon_{i_{t}} \neq 0$ for $t \geqslant 1$. Thus the reflections are admissible.

Now the reflection functors give an equivalence between representations of $\Pi^{\lambda^{(0)}}$ of dimension $\varepsilon_{i}$, of which there is only one, and representations of $\Pi^{\lambda^{(m)}}$ of dimension $\alpha$. Thus there is a unique representation of $\Pi^{\lambda^{(m)}}$ of dimension $\alpha$, up to isomorphism.

Now the restriction of this representation to $Q$ is indecomposable, for if it had an indecomposable direct summand of dimension $\beta$, then by Theorem 3.3 one has $\lambda^{(m)} \cdot \beta=0$. But this is impossible since $\beta$ is a root, not equal to $\pm \alpha$.

Finally, for uniqueness, observe that any indecomposable representation of $Q$ of dimension $\alpha$ lifts to a representation of $\Pi^{\lambda^{(m)}}$ since $\lambda^{(m)} \cdot \alpha=0$. Since there is only one representation of $\Pi^{\lambda^{(m)}}$, it follows that there is only one indecomposable representation of $Q$.

Finally we turn to Kac's Lemma 2. We have an elementary proof of it for indivisible dimension vectors, that is, vectors whose components have no common divisor.

PROPOSITION A.5. If $\alpha \in \mathbb{N}^{I}$ is indivisible then the number of isomorphism classes of indecomposable representations of dimension $\alpha$ (if finite), and the number of parameters $\operatorname{dim}_{\mathrm{G}(\alpha)} I(\alpha)$, are independent of the orientation of $Q$. 
Proof. Since $\alpha$ is indivisible, the general element of $\left\{\lambda \in K^{I} \mid \lambda \cdot \alpha=0\right\}$ has $\lambda \cdot \beta \neq 0$ for all $\beta \in \mathbb{N}^{I}$ with $0<\beta<\alpha$. Choose $\lambda$ with this property. Clearly a representation $x \in \operatorname{Rep}(Q, \alpha)$ lifts to a representation of $\Pi^{\lambda}$ if and only if it is indecomposable. Thus by Lemma 3.4 we have

$$
\operatorname{dim} \mu_{\alpha}^{-1}(\lambda)=\operatorname{dim}_{\mathrm{G}(\alpha)} I(\alpha)+\alpha \cdot \alpha-q(\alpha) .
$$

Moreover, if there are only $m$ isomorphism classes of indecomposables, then $\mu_{\alpha}^{-1}(\lambda)$ is a disjoint union of $m$ irreducible locally closed subsets of dimension $\alpha \cdot \alpha-q(\alpha)$, so it has $m$ irreducible components (the closures of these subsets). Finally it suffices to note that up to isomorphism the scheme $\mu_{\alpha}^{-1}(\lambda)$ does not depend on the orientation of $Q$ (see for example [5, Lemma 2.2]).

Remark A.6. Clearly the proposition holds for general $\alpha$ if one instead uses the set $E(\alpha)$ of representations of dimension $\alpha$ with the property that any direct summand has dimension proportional to $\alpha$. In fact, using these methods it is possible to prove all of Kac's Theorem without using Kac's Lemma 2, except the existence (and number of parameters) of indecomposable representations of dimension $\alpha$ with $\alpha$ a divisible positive root with $q(\alpha)=0$.

\section{References}

1. Bergman, G. M. and Dicks, W.: Universal derivations and universal ring constructions, Pacific J. Math. 79 (1978), 293-337.

2. Cassens, H. and Slodowy, P.: On Kleinian singularities and quivers, Singularities (Oberwolfach, 1996), Birkhäuser, Basel, 1998, pp. 263-288.

3. Crawley-Boevey, W.: Preprojective algebras, differential operators and a Conze embedding for deformations of Kleinian singularities, Comment. Math. Helv. 74 (1999), $548-574$

4. Crawley-Boevey, W.: On the exceptional fibres of Kleinian singularities, Amer. J. Math. 122 (2000), 1027-1037.

5. Crawley-Boevey, W. and Holland, M. P.: Noncommutative deformations of Kleinian singularities, Duke Math. J. 92 (1998), 605-635.

6. Grothendieck, A. and Dieudonné, J.: Eléments de géométrie algébrique IV, part 2, Publ. Math. IHES 24 (1965).

7. Kac, V. G.: Infinite root systems, representations of graphs and invariant theory, Invent. Math. 56 (1980), 57-92.

8. Kac, V. G.: Root systems, representations of quivers and invariant theory, In: F. Gherardelli (ed.), Invariant Theory, Proc. Montecatini 1982, Lecture Notes in Math. 996, Springer, Berlin, 1983, pp. 74-108.

9. King, A. D.: Moduli of representations of finite dimensional algebras, Quart. J. Math. Oxford 45 (1994), 515-530.

10. Kraft, H. and Riedtmann, Ch.: Geometry of representations of quivers, In: P. Webb (ed.), Representations of Algebras, Proc. Durham 1985, London Math. Soc. Lecture Note Series 116, Cambridge Univ. Press, 1986, pp. 109-145.

11. Kronheimer, P. B.: The construction of ALE spaces as hyper-Kähler quotients, $J$. Differential Geom. 29 (1989), 665-683. 
12. Le Bruyn, L. and Procesi, C.: Semisimple representations of quivers, Trans. Amer. Math. Soc. 317 (1990), 585-598.

13. Luna, D.: Slices étales, Bull. Soc. Math. France, Mémoire 33 (1973), 81-105.

14. Lusztig, G.: Quivers, perverse sheaves, and quantized enveloping algebras, J. Amer. Math. Soc. 4 (1991), 365-421.

15. Marsden, J. and Weinstein, A.: Reduction of symplectic manifolds with symmetry, Rep. Math. Phys. 5 (1974), 121-130.

16. Nakajima, H.: Instantons on ALE spaces, quiver varieties, and Kac-Moody algebras, Duke Math. J. 76 (1994), 365-416.

17. Nakajima, H.: Varieties associated with quivers, In: R. Bautista et al. (eds), Representation theory of algebras and related topics, Canadian Math. Soc. Conf. Proc. 19, Amer. Math. Soc., Providence, 1996.

18. Nakajima, H.: Quiver varieties and Kac-Moody algebras, Duke Math. J. 91 (1998), $515-560$.

19. Nakajima, H.: Quiver varieties and finite dimensional representations of quantum affine algebras, J. Amer. Math. Soc. 14 (2001), 145-238.

20. Ringel, C. M.: Tame Algebras and Integral Quadratic Forms, Lecture Notes in Math. 1099, Springer, Berlin, 1984.

21. Rump, W.: Doubling a path algebra, or: how to extend indecomposable modules to simple modules, An. St. Ovidius Constantza 4 (1996), 174-185.

22. Schofield, A.: General representations of quivers, Proc. London Math. Soc. 65 (1992), 46-64.

23. Schofield, A.: Quivers and Kac-Moody Lie algebras, manuscript, 23pp. 